\title{
THE MULTIDIMENSIONAL VAN DER CORPUT TRANSFORMATION
}

\author{
PATRICK SARGOS
}

To the reader: Patrick Sargos was enthusiastically involved in the process of completing this paper when he suddenly and unexpectedly passed away on November 20, 2012. His friends and colleagues thought that his work should be offered to the mathematical community in order to stimulate further research in this field where Sargos was one of the leaders. We have made minor, technical changes where it seemed clear and left void those sections that Sargos had foreseen but did not have time to write up. Where necessary, we inserted footnotes to explain the version retained. We hope that this last paper of Patrick Sargos will be helpful to all mathematicians working in the field. We express here our warm thanks to Joseph A. Vandehey, whose numerous remarks and suggestions have been incorporated and will doubtlessly improve the legibility, and to Ekkehard Krätzel, who gave us his highly valuable overall opinion on the work. We also express our gratitude to all who have helped in the process of making this paper available, in particular Cécile Dartyge, Joël Rivat and Olivier Robert.

Aleksandar Ivić and Gérald Tenenbaum

\begin{abstract}
We make a systematic study of van der Corput's $B$-process for multiple exponential sums. We study directly the important case where the determinant of the Hessian $H_{f}(\mathbf{x})$ of the phase $f$ may be abnormally small. This requires a work on multidimensional stationary phase integrals uniform in $\delta$, the lower bound for $\left|\operatorname{det} H_{f}(\mathbf{x})\right|$. In passing, we obtain an independent result on the asymptotic behaviour of the stationary phase integral when the critical point of the phase is also a singular point of the boundary of the domain of integration. The whole paper is self-contained.
\end{abstract}

Keywords: van der Corput's $B$-process, multidimensional stationary phase integrals, oscillatory integrals, change of variables.

\section{Introduction}

The one dimensional van der Corput's $B$-process goes back to the 1920's. It is the analogue, for exponential sums, of the stationary phase method for oscillatory 
integrals, cf [10]. With the usual notation $e(\xi)=e^{2 i \pi \xi}$, it may be roughly stated as follows:

$$
\begin{aligned}
\sum_{M<m \leqslant 2 M} e\left(T f\left(\frac{m}{M}\right)\right)= & e^{ \pm i \pi / 4} \frac{M}{T^{1 / 2}} \sum_{\alpha \leqslant n / N \leqslant \beta}\left|f^{\prime \prime}\left(\omega\left(\frac{n}{N}\right)\right)\right|^{-1 / 2} e\left(T f^{*}\left(\frac{n}{N}\right)\right) \\
& + \text { Error. }
\end{aligned}
$$

Here, the phase $f:[1,2] \rightarrow \mathbb{R}$ is a four times differentiable function with nonvanishing second derivative; $\omega:[\alpha, \beta] \rightarrow[1,2]$ is the inverse map of $f^{\prime}$; the new phase function $f^{*}$ is defined by $f^{*}(y)=f(\omega(y))-y \omega(y)$, for $y \in[\alpha, \beta]$. Moreover, $M$ and $T$ are large positive real numbers, such that $M<T$, from which we form $N=T / M$, while $m$ and $n$ are integers. Finally, the error term is negligible when $\alpha$ and $\beta$ are not too close from an integer; otherwise, it may be as large as $M / T^{1 / 2}$; a complete and interesting study of this term may be found in [14].

The first exponential sum in (1.1) is trivially bounded by $M$, while the second one is $O\left(T^{1 / 2}\right)$. If we suppose that $T^{1 / 2}$ is smaller than $M$, we deduce at once a non trivial bound:

$$
\sum_{M<m \leqslant 2 M} e\left(T f\left(\frac{m}{M}\right)\right) \ll T^{1 / 2}
$$

called "Van der Corput's inequality". Note that this bound requires only the second derivative of $f$, cf [2].

The analogue of this transformation for two-dimensional exponential sums is not straightforward. It has been initiated by Titchmarsh in 1942; after further improvements, the more precise result to date has been obtained by Krätzel (cf. Theorem 2.24 of [5]; see also the historical notes at the end of chapter 2 in [5]). His statement requires very delicate hypotheses which give rise to a complicated error term. The proof is based on a repeated application of the one-dimensional case.

The same method yields a $p$-dimensional version of van der Corput's transformation, the only correct statement to date (cf [5], Theorem 2.28). However, the error part contains a term whose saving is not better than in the two-dimensional case.

Note that the results of Srinivasan (cf. [11] and [12]) in 1963 and 1965, concerning the multidimensional $B$-process, are probably false because they do not consider enough the role of the boundary of the $p$-dimensional domain of summation; at least, we may assert that the proof is incorrect.

We describe now our version of van der Corput's multidimensional transformation. We consider two cases. We shall call the first one as the "case without boundary", because it concerns exponential sums of the form

$$
\sum_{\mathbf{m} \in \mathbb{Z}^{p}} \chi\left(\frac{m_{1}}{M_{1}}, \ldots, \frac{m_{p}}{M_{p}}\right) e\left(\operatorname{Tf}\left(\frac{m_{1}}{M_{1}}, \ldots, \frac{m_{p}}{M_{p}}\right)\right) .
$$

Here, the phase $f: \Omega \rightarrow \mathbb{R}$ is a $C^{k}$ function defined on a connected bounded open set $\Omega$ of $\mathbb{R}^{p}$ and the weight function $\chi: \mathbb{R}^{p} \rightarrow \mathbb{C}$ is regular with compact support 
in $\Omega$. We suppose that all the derivatives $\partial^{\alpha} f$ and $\partial^{\beta} \chi$, up to some order $k$ are uniformly bounded. The fundamental hypothesis is:

$$
\left|\operatorname{det} H_{f}(\mathbf{x})\right| \geqslant \delta>0 \quad \text { for all } \mathbf{x} \in \Omega \text {, }
$$

where $H_{f}(\mathbf{x})$ is the Hessian of $f$ at $\mathbf{x}$. The number $\delta$ may be small when one applies first the two-dimensional $A$-process, so we want all our derived bounds to be uniform in $\delta$. This will require much more work. Apart from this last point, our result has been implicitly obtained in the proof of Theorem 2.6 of [1], using Hörmander's estimations for stationary phase integrals with nondegenerate critical point (cf. Lemma 4.2 in section 4 below), while the corresponding "van der Corput's inequality" is already implicitly contained in the proof of Theorem 2 of [9] and in previous papers cited there.

Next, we consider the "case with boundary" which concerns exponential sums of the form:

$$
S=\sum_{\substack{\mathbf{m} / \mathbf{M} \in D \\ \mathbf{m} \in \mathbb{Z}^{p}}} \chi\left(\frac{m_{1}}{M_{1}}, \ldots, \frac{m_{p}}{M_{p}}\right) e\left(T f\left(\frac{m_{1}}{M_{1}}, \ldots, \frac{m_{p}}{M_{p}}\right)\right) .
$$

The notations are exactly as in the previous case, except that we have to sum on a compact set $D \subset \Omega$. This may cause great difficulties unless $D$ is very special. We get round this complication with an expedient. So we obtain our sum as a product of two $p$-dimensional exponential sums

$$
S=\int_{[0,1]^{p}} S_{1}(\vartheta) S_{2}(\vartheta) d \vartheta
$$

the first one containing the problem related to the boundary of $D$ and is independent of the phase function $f$, while the second is the one expected after a $B$-process without boundary. Thus, our $B$-process is quite different from Theorem 2.28 of [5]. Even in the one-dimensional case, we do not recover the classical formula (1.1); our error term may be reduced as much as required providing that the number $k$ of derivatives of $f$ is large enough, but our main term is slightly different.

When we have good bounds for $\mu(D)=\int_{[0,1]^{p}}\left|S_{1}(\vartheta)\right| d \vartheta$, in the case $p \geqslant 3$, our Theorem 5.3 becomes an improvement, maybe strong, on corresponding Krätzel's theorem.

\section{Notations and preliminaries}

\subsection{Notations}

In the whole paper, $\Omega$ will denote a connected open set of $\mathbb{R}^{p}, p \geqslant 1$ being a fixed integer.

The expressions $u=O(v)$ and $u \ll v$, with $u \in \mathbb{C}$ and $v>0$, both mean that there exists a constant $C$, such that one has $|u| \leqslant C v$; here, $C$ may depend on other constants which will be recalled at each occurrence whenever a doubt is possible. We shall say that $C$ is an absolute constant if it does not depend on any parameter. 
We shall exclusively consider the euclidean norm in $\mathbb{R}^{p}$, that is $\|\mathbf{x}\|=$ $\left(\sum_{j=1}^{p} x_{j}^{2}\right)^{1 / 2}$, and we shall use the inner product $\langle\mathbf{x}, \mathbf{h}\rangle=\sum_{i=1}^{p} x_{i} h_{i}$.

We shall use the notation $\Omega^{c}$ to define the complementary set of $\Omega$ in $\mathbb{R}^{p}$. For any $\mathbf{x} \in \mathbb{R}^{p}$, the distance of $\mathbf{x}$ to $\Omega^{c}$ will be written as

$$
d\left(\mathbf{x}, \Omega^{c}\right)=\inf _{\substack{\mathbf{y} \in \mathbb{R}^{p} \\ \mathbf{y} \notin \Omega}}\|\mathbf{x}-\mathbf{y}\| .
$$

We shall denote by $M_{p}(\mathbb{R})$ the set of symmetric $p \times p$ non singular real matrices. For any $A \in M_{p}(\mathbb{R})$, its operator norm will be defined as

$$
\|A\|=\sup _{\|\mathbf{x}\| \leqslant 1}\|A \mathbf{x}\|
$$

Let $C^{k}(\Omega, \mathbb{R})$ be the set of functions $f: \Omega \rightarrow \mathbb{R}$ whose partial derivatives up to the order $k$, namely $\partial^{\alpha} f(\mathbf{x})=\partial_{1}^{\alpha_{1}} \ldots \partial_{p}^{\alpha_{p}} f\left(x_{1}, \ldots, x_{p}\right)$ with $\alpha \in \mathbb{N}^{p}$ and $|\alpha|=$ $\alpha_{1}+\ldots+\alpha_{p} \leqslant k$, exist and are continuous on $\Omega .{ }^{1}$ Let $C^{k}(\bar{\Omega}, \mathbb{R})$ be the subset of $C^{k}(\Omega, \mathbb{R})$ of those functions whose partial derivatives up to the order $k$ are the restriction to $\Omega$ of continuous functions on $\bar{\Omega}$, the closure of $\Omega$.

As usual, $f^{\prime}(\mathbf{x})$ will be considered either as a point of $\mathbb{R}^{p}$ or as a linear application, so that we may write $f^{\prime}(\mathbf{x}) \mathbf{h}=\left\langle f^{\prime}(\mathbf{x}), \mathbf{h}\right\rangle$. In the former case, we have the relation

$$
f^{\prime} \in C^{k-1}\left(\Omega, \mathbb{R}^{p}\right)
$$

As for the second derivative of $f$, the matrix associated to the bilinear form $f^{\prime \prime}(\mathbf{x})$ is the Hessian of $f$ at $\mathbf{x}$ :

$$
H_{f}(\mathbf{x})=\left(\begin{array}{cccc}
\partial_{1}^{2} f(\mathbf{x}) & \partial_{2} \partial_{1} f(\mathbf{x}) & \cdots & \partial_{p} \partial_{1} f(\mathbf{x}) \\
\partial_{1} \partial_{2} f(\mathbf{x}) & \partial_{2}^{2} f(\mathbf{x}) & \cdots & \partial_{p} \partial_{2} f(\mathbf{x}) \\
\vdots & \vdots & \ddots & \vdots \\
\partial_{1} \partial_{p} f(\mathbf{x}) & \partial_{2} \partial_{p} f(\mathbf{x}) & \cdots & \partial_{p}^{2} f(\mathbf{x})
\end{array}\right)
$$

In the sequel, $f$ will be the phase function of our exponential sum; because of the fundamental hypothesis (1.4), we may assert that

$$
H_{f}(\mathbf{x}) \in M_{p}(\mathbb{R}), \quad \text { for } \mathbf{x} \in \Omega .
$$

Any $A \in M_{p}(\mathbb{R})$ has exactly $p$ real nonzero eigenvalues $\lambda_{1}, \ldots, \lambda_{p}$ when they are counted with multiplicity. We set

$$
\sigma(A)=\#\left\{i=1, \ldots, p \mid \lambda_{i}>0\right\}-\#\left\{j=1, \ldots, p \mid \lambda_{j}<0\right\} .
$$

\footnotetext{
${ }^{1}$ We draw the reader's attention to the fact that Sargos conforms with the standard French notation according to which $\mathbb{N}$ comprises all non-negative integers.
} 


\subsection{Lemmas on matrices}

We recall some results from general algebra.

Lemma 2.1. Let $A \in M_{p}(\mathbb{R})$, whose eigenvalues with multiplicity are $\lambda_{1}, \ldots, \lambda_{p}$. Then there exists an orthogonal $p \times p$ matrix $P$ such that we have $P^{-1} A P=\Delta$, where $\Delta$ is the diagonal matrix

$$
\Delta=\left(\begin{array}{cccc}
\lambda_{1} & 0 & \cdots & 0 \\
0 & \lambda_{2} & \cdots & 0 \\
\vdots & \vdots & \ddots & \vdots \\
0 & 0 & \cdots & \lambda_{p}
\end{array}\right)
$$

Lemma 2.2. Let $A=\left(a_{i j}\right)_{1 \leqslant i, j \leqslant p} \in M_{p}(\mathbb{R})$. For any $(i, j)$ we denote by $A_{i j}$ the cofactor of $a_{i j}$. We then have

$$
A^{-1}=\left(b_{i j}\right)_{1 \leqslant i, j \leqslant p}, \quad \text { with } b_{i j}=\frac{A_{j i}}{\operatorname{det} A} .
$$

If we suppose furthermore that $\left|a_{i j}\right| \leqslant C$ for any $(i, j)$, then we have the bounds

$$
\left|A_{i j}\right| \leqslant(C \sqrt{p-1})^{p-1}
$$

and

$$
\left\|\left.A^{-1}\left|\| \leqslant p(C \sqrt{p-1})^{p-1}\right| \operatorname{det} A\right|^{-1} .\right.
$$

Proof. Note that (2.8) follows from Hadamard's inequality. Moreover, we have

$$
\begin{aligned}
\left\|A^{-1} \mathbf{x}\right\| & \leqslant\left(\sum_{i=1}^{p}\left(\sum_{j=1}^{p}\left|b_{i j} x_{j}\right|\right)^{2}\right)^{1 / 2} \leqslant C_{0} \sqrt{p} \sum_{j=1}^{p}\left|x_{j}\right| \\
& \leqslant C_{0} p\|\mathbf{x}\|, \text { with } C_{0}=\max _{i, j}\left|b_{i j}\right|,
\end{aligned}
$$

by Cauchy's inequality, and this is (2.9).

\subsection{An extension lemma for continuous functions}

The following result from general analysis will simplify our exposition at several occasions.

Lemma 2.3. Let $F: V \rightarrow E$ be a mapping from a subset $V$ of a normed space $E_{0}$ into a complete normed space $E_{\widetilde{F}}$. Assume that $F$ is uniformly continuous on $V$. Then there exists an extension $\widetilde{F}$ of $F$ to a continuous mapping of $\bar{V}$ into $E$.

This is Theorem 2.6 of chapter VII of [7]. We shall use it when $V$ is a convex open subset of $\mathbb{R}^{p}$, and $F$ is a $C^{1}$ function whose partial derivatives are bounded in $V$. 


\subsection{A two-dimensional change of variables}

Let $c$ be a positive real number, and $\varphi_{1}$ and $\varphi_{2}$ be two functions in $C^{k}(] 0, c\left[^{2}, \mathbb{R}\right)$ for some integer $k \geqslant 2$. We suppose that we have, for $\nu=1,2$,

$$
\begin{array}{rlrl}
\lim _{r \rightarrow 0^{+}, s \rightarrow 0^{+}} \varphi_{\nu}(r, s)=0 \quad \text { and } & \left|\partial^{\alpha} \varphi_{\nu}(r, s)\right| & \leqslant Z^{|\alpha|} \\
\text { for } 1 & \leqslant|\alpha| \leqslant k,(r, s) \in] 0, c\left[^{2}\right.
\end{array}
$$

for some $Z>0$.

We want to show that, assuming that $c$ is small enough, the relation

$$
\left\{\begin{array}{l}
x=r\left(1+\varphi_{1}(r, s)\right) \\
y=s\left(1+\varphi_{2}(r, s)\right)
\end{array}\right.
$$

defines a two-dimensional change of variables. We state this in a precise form.

Lemma 2.4. If we assume $c \leqslant 1 /(6 Z)$, then the mapping $F$ defined by

$$
F(r, s)=\left(\begin{array}{l}
x(r, s) \\
y(r, s)
\end{array}\right)=\left(\begin{array}{l}
r\left(1+\varphi_{1}(r, s)\right) \\
s\left(1+\varphi_{2}(r, s)\right)
\end{array}\right) \quad \text { for }(r, s) \in V
$$

is a $C^{k}$-diffeomorphism from $\left.V=\right] 0, c\left[^{2}\right.$ onto $W=F(V) \subset \mathbb{R}^{2}$, whose inverse map $F^{-1}$ may be written as

$$
F^{-1}(x, y)=\left(\begin{array}{l}
r(x, y) \\
s(x, y)
\end{array}\right)=\left(\begin{array}{l}
x\left(1+w_{1}(x, y)\right) \\
y\left(1+w_{2}(x, y)\right)
\end{array}\right) \quad \text { for }(x, y) \in W
$$

where, for $\nu=1,2$, the function $w_{\nu}$ is in $C^{k-1}(W, \mathbb{R})$ and satisfies

$$
\begin{array}{r}
\lim _{x \rightarrow 0^{+}, y \rightarrow 0^{+}} w_{\nu}(x, y)=0 \quad \text { and } \quad \partial^{\alpha} w_{\nu}(x, y) \ll Z^{|\alpha|} \\
\quad \text { for }(x, y) \in W \quad \text { and } 1 \leqslant|\alpha| \leqslant k-1,
\end{array}
$$

the implied constant depending only on $k$. Moreover, we have the inclusion

$$
] 0, c / 2\left[^{2} \subset W \subset\right] 0,+\infty\left[^{2} .\right.
$$

Proof. a) We first prove the injectivity of $F$ when $c$ is small enough. For this, we suppose that we have

$$
F(r+h, s+k)=F(r, s)
$$

for some $(r, s)$ and $(r+h, s+k)$ in $V$, with $(h, k) \neq(0,0)$. The two-dimensional mean value theorem applied to $x$ and $y$ yields the equality

$$
\left\{\begin{array}{l}
0=x(r+h, s+k)-x(r, s)=\left\langle x^{\prime}\left(r+\vartheta_{1} h, s+\vartheta_{1} k\right),(h, k)\right\rangle \\
0=y(r+h, s+k)-y(r, s)=\left\langle y^{\prime}\left(r+\vartheta_{2} h, s+\vartheta_{2} k\right),(h, k)\right\rangle
\end{array}\right.
$$


which holds for some $\left.\left(\vartheta_{1}, \vartheta_{2}\right) \in\right] 0,1\left[^{2}\right.$. This means that the linear system

$$
\left(\begin{array}{ll}
\frac{\partial x}{\partial r}\left(r_{1}, s_{1}\right) & \frac{\partial x}{\partial s}\left(r_{1}, s_{1}\right) \\
\frac{\partial y}{\partial r}\left(r_{2}, s_{2}\right) & \frac{\partial y}{\partial s}\left(r_{2}, s_{2}\right)
\end{array}\right)\left(\begin{array}{l}
h \\
k
\end{array}\right)=(0,0)
$$

with $\left(r_{\nu}, s_{\nu}\right)=\left(r+\vartheta_{\nu} h, s+\vartheta_{\nu} k\right)$ for $\nu=1,2$, admits a non trivial solution $(h, k)$, and thus its determinant is zero.

We are going to prove that this is impossible. For simplification, we write $u, \frac{\partial u}{\partial r}$, etc., instead of $u(r, s), \frac{\partial u}{\partial r}(r, s)$, etc. We note the inequalities

$$
|h|,|k| \leqslant c ; \quad 0 \leqslant r, s \leqslant c ; \quad\left|\varphi_{\nu}\right| \leqslant 2 c Z \leqslant 1 / 3, \quad \text { for } \quad \nu=1,2,
$$

the latter being a consequence of the mean value theorem, and of the limit

$$
\lim _{r \rightarrow 0^{+}, s \rightarrow 0^{+}} \varphi_{\nu}(r, s)=0 .
$$

The assumption $c \leqslant(6 Z)^{-1}$ ensures that

$$
\frac{\partial x}{\partial r}=1+\varphi_{1}+r \frac{\partial \varphi_{1}}{\partial r} \geqslant 1 / 2, \quad \text { and } \quad \frac{\partial y}{\partial s}=1+\varphi_{2}+s \frac{\partial \varphi_{2}}{\partial s} \geqslant 1 / 2
$$

on the one hand, and

$$
\left|\frac{\partial x}{\partial s}\right|=\left|r \frac{\partial \varphi_{1}}{\partial s}\right| \leqslant 1 / 6 \quad \text { and } \quad\left|\frac{\partial y}{\partial r}\right|=\left|s \frac{\partial \varphi_{2}}{\partial r}\right| \leqslant 1 / 6,
$$

on the other hand. This shows that the absolute value of the determinant of the $2 \times 2$ above matrix is $\geqslant 1 / 4-1 / 36=2 / 9>0$. We thus have proved the injectivity of $F$.

b) We know now that $F$ is bijective from $V$ onto $W$. The Jacobian matrix of $F$ is

$$
J_{F}(r, s)=\left(\begin{array}{ll}
\frac{\partial x}{\partial r}(r, s) & \frac{\partial x}{\partial s}(r, s) \\
\frac{\partial y}{\partial r}(r, s) & \frac{\partial y}{\partial s}(r, s)
\end{array}\right) .
$$

Its determinant is $\Delta=\Delta(r, s)=1+u=1+u(r, s)$, with

$$
u=\varphi_{1}+\varphi_{2}+r \partial_{1} \varphi_{1}+s \partial_{2} \varphi_{2}+\left(\varphi_{1}+r \partial_{1} \varphi_{1}\right)\left(\varphi_{2}+s \partial_{2} \varphi_{2}\right)-r s \partial_{2} \varphi_{1} \partial_{1} \varphi_{2},
$$

and we have, using the same proof as above, $\Delta \geqslant 2 / 9$.

Now, we write the matrix equality $J_{F^{-1}}=\left(J_{F}\right)^{-1}$ to get

$$
\left(\begin{array}{ll}
\frac{\partial r}{\partial x} & \frac{\partial r}{\partial y} \\
\frac{\partial s}{\partial x} & \frac{\partial s}{\partial y}
\end{array}\right)=\frac{1}{\Delta}\left(\begin{array}{cc}
\frac{\partial y}{\partial s} & -\frac{\partial x}{\partial s} \\
-\frac{\partial y}{\partial r} & \frac{\partial x}{\partial r}
\end{array}\right)
$$

from which we deduce

$$
\left\{\begin{array}{l}
\frac{\partial r}{\partial x}(x, y)=\frac{1}{\Delta(r(x, y), s(x, y))} \frac{\partial y}{\partial s}(r(x, y), s(x, y)) \\
\frac{\partial r}{\partial y}(x, y)=\frac{-1}{\Delta(r(x, y), s(x, y))} \frac{\partial x}{\partial s}(r(x, y), s(x, y)) \\
\frac{\partial s}{\partial x}(x, y)=\frac{-1}{\Delta(r(x, y), s(x, y))} \frac{\partial y}{\partial r}(r(x, y), s(x, y)) \\
\frac{\partial s}{\partial y}(x, y)=\frac{1}{\Delta(r(x, y), s(x, y))} \frac{\partial x}{\partial r}(r(x, y), s(x, y))
\end{array} .\right.
$$


We see that the partial derivatives of order one of $r(x, y)$ and $s(x, y)$ may be expressed in terms of $r, s, \varphi_{1}, \varphi_{2}$ and of the partial derivatives of order one of $\varphi_{1}$ and $\varphi_{2}$, and are $O(1)$. We thus have proved that $F^{-1}$ is in $C^{1}\left(W, \mathbb{R}^{2}\right)$.

Deriving (2.17), we show successively that $F^{-1}$ is in $C^{l}\left(W, \mathbb{R}^{2}\right)$, for $l=$ $2,3, \ldots, k$. and we get the bound

$$
\begin{array}{rl}
\partial^{\alpha} r(x, y) \ll Z^{|\alpha|-1} \quad \text { and } \quad \partial^{\alpha} s(x, y) & \ll Z^{|\alpha|-1}, \\
\text { for } 1 & 1 \leqslant|\alpha| \leqslant k \text { and }(x, y) \in W .
\end{array}
$$

c) We are now going to prove (2.12) and (2.13), and it is sufficient to do this for $r$ only, the case of $s$ being identical. As $\left|\varphi_{\nu}(r, s)\right| \leqslant 1 / 3$ according to (2.14), and because of the definition of $x(r, s)$ and $y(r, s)$, given in $(2.11)$, we have obviously the inclusion (2.14); the same arguments show that we have the limits

$$
\lim _{x \rightarrow 0^{+}, y \rightarrow 0^{+}} r(x, y)=\lim _{x \rightarrow 0^{+}, y \rightarrow 0^{+}} s(x, y)=0 .
$$

By the mean value theorem in Lagrange's form, we have

$$
r(x, y)=x \int_{0}^{1} \frac{\partial r}{\partial x}(t x, t y) d t+y \int_{0}^{1} \frac{\partial r}{\partial y}(t x, t y) d t .
$$

Using (2.16) and (2.17), we see that

$$
\int_{0}^{1} \frac{\partial r}{\partial x}(t x, t y) d t=1+v(x, y)
$$

and

$$
\int_{0}^{1} \frac{\partial r}{\partial y}(t x, t y) d t=x w(x, y)
$$

where $v$ and $w$ are $C^{k-1}$ functions on $W$ such that

$$
\partial^{|\alpha|} v(x, y) \ll Z^{|\alpha|} \quad \text { and } \quad \partial^{|\alpha|} w(x, y) \ll Z^{|\alpha|+1},
$$

for $1 \leqslant|\alpha| \leqslant k-1$ and $(x, y) \in W$.

We set

$$
w_{1}(x, y)=v(x, y)+y w(x, y)
$$

so that $w_{1}$ satisfies the second part of (2.13). But for the first part of (2.13), this is only a problem of limit, and not of differential calculus, which is an easy consequence of (2.11), (2.12) and the first part of (2.10). The lemma is entirely proved.

\subsection{A special one-dimensional oscillatory integral}

Let $\chi:\left[0,+\infty\left[\rightarrow \mathbb{C}\right.\right.$ be a $C^{p+1}$ function which satisfies

$$
\left|\chi^{(j)}(x)\right| \leqslant Z^{j}, \quad \text { for } 0 \leqslant j \leqslant 2 \text { and } x \geqslant 0,
$$


and

$$
\chi(x)=0 \quad \text { for } \quad x \geqslant c
$$

where $Z$ and $c$ are real parameters satisfying

$$
0<c \leqslant 1 \leqslant Z \text {. }
$$

We want to determine the asymptotic behavior, with error terms effective in $c$ and $Z$, of the oscillatory integral

$$
J_{p}(\tau)=\int_{0}^{\infty} \chi(x) x^{p-1} e\left(\tau x^{2}\right) d x
$$

for large values of the real parameter $\tau$, while $p$ is a fixed positive integer.

Lemma 2.5. With the above notations, we suppose that

$$
\tau \geqslant c^{-2}+c^{2} Z^{4}
$$

Then we have

$$
J_{p}(\tau)=\frac{\Gamma(p / 2)}{2(2 \pi)^{p / 2}} \chi(0) e^{p i \pi / 4} \tau^{-p / 2}+O\left(\frac{c^{-1}+c Z^{2}}{\tau^{(p+1) / 2}}\right),
$$

where the above implied constant depends at most on $p$, and where $\Gamma$ denotes Euler's Gamma function.

Proof. a) Along the proof of this lemma, the main step is an integration by parts which occurs a lot of times. More precisely, let $q \geqslant 1$ be an integer. We have

$$
\chi(x) x^{q} e\left(\tau x^{2}\right)=\frac{\chi(x) x^{q-1}}{4 i \pi \tau} \frac{d}{d x}\left(e\left(\tau x^{2}\right)\right),
$$

so that we deduce, for $q \geqslant 2$,

$$
\int_{0}^{\infty} \chi(x) x^{q} e\left(\tau x^{2}\right) d x=\frac{-1}{4 i \pi \tau} \int_{0}^{\infty}\left((q-1) \chi(x) x^{q-2}+\chi^{\prime}(x) x^{q-1}\right) e\left(\tau x^{2}\right) d x,
$$

and

$$
\int_{0}^{\infty} \chi(x) x^{q} e\left(\tau x^{2}\right) d x=-\frac{\chi(0)}{4 i \pi \tau}-\frac{1}{4 i \pi \tau} \int_{0}^{\infty} \chi^{\prime}(x) e\left(\tau x^{2}\right) d x,
$$

for $q=1$.

b) Now, we deal with the case $p=1$. We write

$$
\begin{aligned}
J_{1}(\tau) & =\int_{0}^{c} \chi(x) e\left(\tau x^{2}\right) d x \\
& =\chi(0) \int_{0}^{c} e\left(\tau x^{2}\right) d x+\int_{0}^{c}(\chi(x)-\chi(0)) e\left(\tau x^{2}\right) d x .
\end{aligned}
$$


For the first integral, we have

$$
\begin{aligned}
\int_{0}^{c} e\left(\tau x^{2}\right) d x & =\int_{0}^{\infty} e\left(\tau x^{2}\right) d x-\int_{c}^{\infty} e\left(\tau x^{2}\right) d x \\
& =\frac{e^{i \pi / 4}}{2^{3 / 2} \tau^{1 / 2}}+O\left(\frac{1}{c \tau}\right) .
\end{aligned}
$$

For the last integral in $(2.27)$, we first note that $\chi(x)-\chi(0)=\int_{0}^{1} \chi^{\prime}(t x) x d t$. We thus have

$$
\int_{0}^{c}(\chi(x)-\chi(0)) e\left(\tau x^{2}\right) d x=\int_{0}^{1}\left(\int_{0}^{c} \chi^{\prime}(t x) x e\left(\tau x^{2}\right) d x\right) d t .
$$

For bounding the inner integral, we integrate by parts:

$$
\begin{aligned}
\int_{0}^{c} \chi^{\prime}(t x) x e\left(\tau x^{2}\right) d x & =\frac{\chi^{\prime}(t c) e\left(t c^{2}\right)-\chi^{\prime}(0)}{4 i \pi \tau}-\frac{t}{4 i \pi \tau} \int_{0}^{c} \chi^{\prime \prime}(t x) e\left(\tau x^{2}\right) d x \\
& \ll \frac{Z}{\tau}+\frac{c Z^{2}}{\tau}
\end{aligned}
$$

Now, we insert (2.28) and this last bound in (2.27) to get (2.25) in the case $p=1$.

c) We now deal with the case $p=2$. An integration by parts yields

$$
\begin{aligned}
J_{2}(\tau) & =\int_{0}^{\infty} \chi(x) x e\left(\tau x^{2}\right) d x=-\frac{\chi(0)}{4 i \pi \tau}-\frac{1}{4 i \pi \tau} \int_{0}^{\infty} \chi^{\prime}(x) e\left(\tau x^{2}\right) d x \\
& =\frac{\chi(0) e^{2 i \pi / 4}}{4 \pi \tau}+O\left(\frac{Z}{\tau^{3 / 2}}\right)
\end{aligned}
$$

the last bound being due to (2.25) in the case $p=1$, completed with (2.24); we note that the second derivative of $\chi$ is indeed needed. We have proved (2.25) for $p=2$.

d) We conclude the general case by a recurrence relation, deduced from an integration by parts. More precisely, we have, for $p \geqslant 3$

$$
J_{p}(\tau)=\frac{-(p-2)}{4 i \pi \tau} \int_{0}^{\infty} \chi(x) x^{p-3} e\left(\tau x^{2}\right) d x-\frac{1}{4 i \pi \tau} \int_{0}^{\infty} \chi^{\prime}(x) x^{p-2} e\left(\tau x^{2}\right) d x .
$$

From this recurrence formula, and the cases $p=1$ or $p=2$, we deduce the expected result.

Remark. Separating the real and the imaginary part of $\chi$, and taking then the complex conjugate of $(2.25)$, we get

$$
\int_{0}^{\infty} \chi(x) x^{p-1} e\left(-\tau x^{2}\right) d x=\frac{\Gamma(p / 2)}{2(2 \pi)^{p / 2}} \chi(0) e^{-p i \pi / 4} \tau^{-p / 2}+O\left(\frac{c^{-1}+c Z^{2}}{\tau^{(p+1) / 2}}\right) .
$$




\subsection{A special two-dimensional oscillatory integral}

Let $k, m, n, p$ be positive integers, with

$$
m+n=p, \quad k=p+4 .
$$

Let $c$ and $Z$ be real parameters satisfying $(2.22)$ and let $\chi \in C^{k}\left(\left[0,+\infty\left[^{2}, \mathbb{C}\right)\right.\right.$, which satisfies

$$
\begin{cases}\left|\partial^{\alpha} \chi(x, y)\right| \leqslant Z^{|\alpha|}, & \text { for } 0 \leqslant|\alpha| \leqslant k \text { and }(x, y) \in] 0,+\infty\left[{ }^{2} .\right. \\ \chi(x, y)=0, & \text { if } x \geqslant c \text { or if } y \geqslant c .\end{cases}
$$

We want to determine the asymptotic behavior, with explicit error terms, of the two-dimensional oscillatory integral

$$
J_{m, n}(\tau)=\int_{0}^{\infty} \int_{0}^{\infty} \chi(x, y) x^{m-1} y^{n-1} e\left(\tau\left(x^{2}-y^{2}\right)\right) d x d y
$$

where $\tau$ is a real parameter such that

$$
\tau \geqslant \mu\left(c^{-2}+c^{2} Z^{4}\right)
$$

for some constant $\mu>0$. At last, we set $\sigma=m-n$.

Lemma 2.6. With the above notation, there exists a constant $\mu>0$ which depends only on $k$, such that, under the assumption (2.37), we have

$$
J_{m, n}(\tau)=\frac{\Gamma(m / 2) \Gamma(n / 2)}{4(2 \pi)^{p / 2}} \chi(0,0) e^{i \sigma \pi / 4} \tau^{-p / 2}+O\left(\frac{c^{-1}+Z}{\tau^{(p+1) / 2}}\right),
$$

where the implied constant depends at most on $k$.

Proof. a) We fix a $C^{\infty}$ function $\varrho:[0,+\infty[\rightarrow \mathbb{R}$ which satisfies

$$
\begin{cases}\varrho(x)=1 & \text { if } 0 \leqslant x \leqslant c \\ 0 \leqslant \varrho(x) \leqslant 1 & \text { if } c \leqslant x \leqslant 2 c \\ \varrho(x)=0 & \text { if } x \geqslant 2 c\end{cases}
$$

and so that

$$
\left|\varrho^{(j)}(x)\right| \leqslant L c^{-j}, \quad \text { for } x \geqslant 0 \text { and } 1 \leqslant j \leqslant k,
$$

for some constant $L>0$, depending only on $k$.

Applying Taylor's formula up to the first order to $\chi$, we get

$$
\chi(x, y)=\varrho(x) \varrho(y) \chi(0,0)+x \chi_{1}(x, y)+y \chi_{2}(x, y)
$$

with

$$
\chi_{j}(x, y)=\varrho(x) \varrho(y) \int_{0}^{1} \partial_{j} \chi(t x, t y) d t, \quad j=1,2 .
$$


Inserting this in (2.36), we get $J_{m, n}(\tau)$ as the sum of three terms. The first one is

$$
\chi(0,0)\left(\int_{0}^{\infty} \varrho(x) x^{m-1} e\left(\tau x^{2}\right) d x\right)\left(\int_{0}^{\infty} \varrho(y) y^{n-1} e\left(-\tau y^{2}\right) d y\right)
$$

and, according to Lemma 2.5, this is equal to

$$
\frac{\Gamma(m / 2) \Gamma(n / 2)}{4(2 \pi)^{p / 2}} \chi(0,0) e^{\sigma i \pi / 4} \tau^{-p / 2}+O\left(\frac{c^{-1}}{\tau^{(p+1) / 2}}\right),
$$

providing that $\tau \gg c^{-2}$, for suitable implied constants depending at most on $k$.

The two other terms are

$$
I_{1}=\int_{0}^{\infty} \int_{0}^{\infty} \chi_{1}(x, y) x^{m} y^{n-1} e\left(\tau\left(x^{2}-y^{2}\right)\right) d x d y
$$

and

$$
I_{2}=\int_{0}^{\infty} \int_{0}^{\infty} \chi_{2}(x, y) x^{m-1} y^{n} e\left(\tau\left(x^{2}-y^{2}\right)\right) d x d y .
$$

We have thus obtained the formula:

$$
J_{m, n}(\tau)=\frac{\Gamma(m / 2) \Gamma(n / 2)}{4(2 \pi)^{p / 2}} \chi(0,0) e^{\sigma i \pi / 4} \tau^{-p / 2}+I_{1}+I_{2}+O\left(\frac{c^{-1}}{\tau^{(p+1) / 2}}\right) .
$$

It remains now to give upper bounds for $I_{1}$ and $I_{2}$. Because of the symmetry between the two terms, it suffices, for instance, to prove that we have

$$
I_{1} \ll \frac{Z}{\tau^{(p+1) / 2}} .
$$

b) We are going to write $I_{1}$ as a one-dimensional oscillatory integral, using Lemma 2.4. Indeed, we have

$$
\begin{aligned}
I_{1} & =\int_{0}^{\infty} y^{n-1} e\left(-\tau y^{2}\right)\left(\int_{0}^{\infty} \chi_{1}(x, y) x^{m} e\left(\tau x^{2}\right) d x\right) d y \\
& =Z \tau^{-(m+1) / 2} \int_{0}^{\infty} F_{\tau}(y) y^{n-1} e\left(-\tau y^{2}\right) d y
\end{aligned}
$$

where we have set

$$
F_{\tau}(y)=\frac{\tau^{(m+1) / 2}}{Z} \int_{0}^{\infty} \chi_{1}(x, y) x^{m} e\left(\tau x^{2}\right) d x .
$$

As we have

$$
F_{\tau}^{(j)}(y)=\frac{\tau^{(m+1) / 2}}{Z} \int_{0}^{\infty} \partial_{2}^{j} \chi_{1}(x, y) x^{m} e\left(\tau x^{2}\right) d x
$$


we apply Lemma 2.5 to get

$$
F_{\tau}^{(j)}(y) \ll c^{-j}+Z^{j}, \quad \text { for } 0 \leqslant j \leqslant k-m-3 \text { and } y \geqslant 0,
$$

the implied constant depending at most on $k$.

We note that the proof of Lemma 2.5 does not require that the weight function $\chi$ be independent of $\tau$, provided that the upper bounds for its derivatives are uniform. So that we may apply $(2.30)$ to the integral $(2.45)$ with the weight function $F_{\tau}$, and this yields

$$
\int_{0}^{\infty} F_{\tau}(y) y^{n-1} e\left(-\tau y^{2}\right) d y=\frac{\Gamma(n / 2)}{2(2 \pi)^{n / 2}} F_{\tau}(0) e^{-n i \pi / 4} \tau^{-n / 2}+O\left(\frac{c^{-1}+c Z^{2}}{\tau^{(n+1) / 2}}\right),
$$

provided that $\tau \gg\left(c^{-2}+c^{2} Z^{4}\right)$, for suitable implied constants that depend only on $k$. This equality yields the bound

$$
\int_{0}^{\infty} F_{\tau}(y) y^{n-1} e\left(-\tau y^{2}\right) d y \ll \tau^{-n / 2}+\frac{c^{-1}+c Z^{2}}{\tau^{(n+1) / 2}} .
$$

We insert this in (2.45) to get

$$
I_{1} \ll \frac{Z}{\tau^{(p+1) / 2}}+\frac{Z\left(c^{-1}+c Z^{2}\right)}{\tau^{p / 2+1}} \ll \frac{Z}{\tau^{(p+1) / 2}},
$$

according to (2.34). We have proved (2.44) and the lemma follows.

\section{The function $f^{*}$}

\subsection{Elementary properties}

Let $\Omega$ be a connected open set of $\mathbb{R}^{p}$, and let $f \in C^{k}(\Omega, \mathbb{R})$, with $k \geqslant 2$. We suppose that $H_{f}(\mathbf{x})$ is a regular matrix for each $\mathbf{x} \in \Omega$. In fact, we suppose that, for some constant $\delta \in] 0,1]$, we have

$$
\left|\operatorname{det} H_{f}(\mathbf{x})\right| \geqslant \delta>0, \quad \mathbf{x} \in \Omega,
$$

as announced in the introduction. Thus $U:=f^{\prime}(\Omega)$ is a connected open set of $\mathbb{R}^{p}$. We make the hypothesis that

$$
f^{\prime}: \Omega \rightarrow U \quad \text { is injective. }
$$

Let $\omega: U \rightarrow \Omega$ denote the inverse map of $f^{\prime}$; we set

$$
f^{*}(\mathbf{y})=f(\omega(\mathbf{y}))-\langle\mathbf{y}, \omega(\mathbf{y})\rangle, \quad \mathbf{y} \in U .
$$

We thus have the diagram

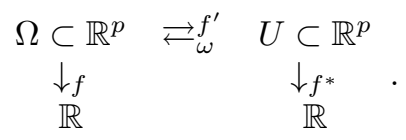

Now, we sum up the more elementary properties of $f^{*}$. 


\section{Proposition 3.1.}

a) $f^{*}: U \rightarrow \mathbb{R}$ is a $C^{k}$ function and we have

$$
\left(f^{*}\right)^{\prime}(\mathbf{y})=-\omega(\mathbf{y}) \quad \text { and } \quad H_{f^{*}}(\mathbf{y})=H_{f}(\omega(\mathbf{y}))^{-1}
$$

for all $y \in U$.

b) $\left(f^{*}\right)^{*}(\mathbf{x})=f(-\mathbf{x})(\mathbf{x} \in \Omega)$.

c) We suppose that the following bound holds

$$
\left|\partial^{\alpha} f(\mathbf{x})\right| \leqslant C, \quad \text { for } 2 \leqslant|\alpha| \leqslant k \text { and } \mathbf{x} \in \Omega .
$$

We then have

$$
\left|\partial^{\alpha} f^{*}(\mathbf{y})\right| \ll \delta^{3-2|\alpha|}, \quad \text { for } 2 \leqslant|\alpha| \leqslant k \text { and } \mathbf{y} \in U .
$$

The constant implied in the $\ll$ depends at most on $C, k$ and $p$.

Proof. The proofs of a) and b) may be deduced from the definitions of $f^{*}$ and $\omega$. If $|\alpha|=2$, then (3.6) is a consequence of (2.9) and (3.4). If, for instance, we differentiate $f^{*}$ three times, then, according to (3.4), we have to differentiate the coefficients of $H_{f}(\omega(\mathbf{y}))^{-1}$; it suffices thus to apply (2.7). Higher order derivatives are obtained similarly.

\subsection{Calculation of $(f+\eta u)^{*}$ when $\eta$ is small}

Let $f \in C^{k}(\Omega, \mathbb{R})$, satisfying (3.1), (3.2), (3.3) and (3.5), and we suppose furthermore that $k \geqslant 4$. Let $u \in C^{k}(\Omega, \mathbb{R})$ satisfying

$$
\left|\partial^{\alpha} u(\mathbf{x})\right| \leqslant C, \quad \text { for } 1 \leqslant|\alpha| \leqslant k \text { and } \mathbf{x} \in \Omega .
$$

Let now $\eta>0$ be a small real number. We set

$$
f_{\eta}(\mathbf{x})=f(\mathbf{x})+\eta u(\mathbf{x}), \quad U_{\eta}=f_{\eta}^{\prime}(\Omega) \text { and } V=U \cap U_{\eta} .
$$

We assume that

$f_{\eta}^{\prime}$ is injective, $\quad V \neq \emptyset, \quad$ and $\quad\left|\operatorname{det} H_{f_{\eta}}(\mathbf{x})\right| \geqslant \delta, \quad$ for $\mathbf{x} \in \Omega$

so that we may define $f_{\eta}^{*}$.

Proposition 3.2. With the above hypotheses, there exists a constant $C_{1}>1$, which depends at most on $p, k$ and $C$, so that, if $\eta \leqslant \delta / C_{1}$, the two following equalities hold for $\mathbf{y} \in V$ :

$$
f_{\eta}^{*}(\mathbf{y})=f^{*}(\mathbf{y})+\eta u(\omega(\mathbf{y}))+\eta^{2} \varphi_{2}(\mathbf{y})
$$

and

$$
\begin{aligned}
f_{\eta}^{*}(\mathbf{y})= & f^{*}(\mathbf{y})+\eta u(\omega(\mathbf{y})) \\
& -\frac{\eta^{2}}{2}\left\langle\left(H_{f}(\omega(\mathbf{y}))^{-1} u^{\prime}(\omega(\mathbf{y})), u^{\prime}(\omega(\mathbf{y}))\right\rangle+\eta^{3} \varphi_{3}(\mathbf{y}) .\right.
\end{aligned}
$$


Here, for $j=2$ or $3, \varphi_{j}$ is in $C^{k-j}(V, \mathbb{R})$ and satisfies

$$
\left|\partial^{\alpha} \varphi_{j}(\mathbf{y})\right| \ll \delta^{3-2 j-2|\alpha|}, \quad \text { for } 0 \leqslant|\alpha| \leqslant k-j, \text { and } \mathbf{y} \in V \text {. }
$$

The constant implied in the above $\ll$ symbol depends at most on $p, k$ and $C$.

Proof. a) First of all, we recall the forms in which we shall use Taylor's formula. Let $W$ be a convex open set of $\mathbb{R}^{p}$ and let $\Phi \in C^{r}\left(W, \mathbb{R}^{q}\right)$, with $r \geqslant 2$ and $q \geqslant 1$. Let $\mathbf{a}$ and $\mathbf{a}+\mathbf{h}$ be two points in $W$. We then have

$$
\Phi(\mathbf{a}+\mathbf{h})=\Phi(\mathbf{a})+\sum_{i=1}^{p} h_{i} \int_{0}^{1} \partial_{i} \Phi(\mathbf{a}+t \mathbf{h}) d t
$$

and

$$
\Phi(\mathbf{a}+\mathbf{h})=\Phi(\mathbf{a})+\Phi^{\prime}(\mathbf{a}) \mathbf{h}+\sum_{i, j=1}^{p} h_{i} h_{j} \int_{0}^{1}(1-t) \partial_{i} \partial_{j} \Phi(\mathbf{a}+t \mathbf{h}) d t .
$$

Here, we have set $\Phi^{\prime}(\mathbf{a}) \mathbf{h}=\sum_{i=1}^{p} h_{i} \partial i \Phi(\mathbf{a}) \in \mathbb{R}^{q}$, so that the equalities (3.13) and (3.14) hold in $\mathbb{R}^{q}$.

b) Let $\omega_{\eta}$ denote the reciprocal of $f_{\eta}^{\prime}$ and let $v(\mathbf{y})=\omega_{\eta}(\mathbf{y})-\omega(\mathbf{y})$, so that $v \in C^{k-1}\left(V, \mathbb{R}^{p}\right)$. Our next goal is to give bounds for $v$ and for its derivatives. For this, we write

$$
\mathbf{y}=f^{\prime}(\omega(\mathbf{y}))=f_{\eta}^{\prime}(\omega(\mathbf{y}))-\eta u^{\prime}(\omega(\mathbf{y}))
$$

and thus

$$
\omega_{\eta}(\mathbf{y})=\omega_{\eta}\left(f_{\eta}^{\prime}(\omega(\mathbf{y}))-\eta u^{\prime}(\omega(\mathbf{y}))\right)
$$

Now, we apply (3.13):

$$
v(\mathbf{y})=-\eta \sum_{i=1}^{p} \partial_{i} u(\omega(\mathbf{y})) \int_{0}^{1} \partial_{i} \omega_{\eta}\left(f_{\eta}^{\prime}(\omega(\mathbf{y}))-\eta t u^{\prime}(\omega(\mathbf{y}))\right) d t .
$$

From this, we get

$$
\left\|\partial^{\alpha} v(\mathbf{y})\right\| \ll \eta \delta^{-1-2|\alpha|}, \quad \text { for } 0 \leqslant|\alpha| \leqslant k-2,
$$

where the implied constant depends at most on $C, k$ and $p$. Note that (3.17) for $\alpha=\mathbf{0}$ is deduced from the bound

$$
\left\|\partial_{i} \omega_{\eta}(\xi)\right\| \ll \delta^{-1}, \quad \text { for } \xi \in V
$$

which follows from (3.4) and (2.7), and similarly for the other values of $\alpha$ in deriving (3.16).

c) We now aim at proving (3.10). We write

$$
\begin{aligned}
f_{\eta}^{*}(\mathbf{y}) & =f_{\eta}\left(\omega_{\eta}(\mathbf{y})\right)-\left\langle\mathbf{y}, \omega_{\eta}(\mathbf{y})\right\rangle \\
& =f(\omega(\mathbf{y})+v(\mathbf{y}))+\eta u(\omega(\mathbf{y})+v(\mathbf{y}))-\langle\mathbf{y}, \omega(\mathbf{y})+v(\mathbf{y})\rangle \\
& =f^{*}(\mathbf{y})+\eta u(\omega(\mathbf{y}))+\eta^{2} \varphi_{2}(\mathbf{y}),
\end{aligned}
$$


where we have set

$$
\begin{aligned}
\varphi_{2}(\mathbf{y})= & \eta^{-2} \sum_{i, j=1}^{p} v_{i}(\mathbf{y}) v_{j}(\mathbf{y}) \int_{0}^{1}(1-t) \partial_{i} \partial_{j} f(\omega(\mathbf{y})+t v(\mathbf{y})) d t \\
& +\eta^{-1} \sum_{i=1}^{p} v_{i}(\mathbf{y}) \int_{0}^{1} \partial_{i} u(\omega(\mathbf{y})+t v(\mathbf{y})) d t
\end{aligned}
$$

with the notation

$$
v(\mathbf{y})=\left(v_{1}(\mathbf{y}), \ldots, v_{p}(\mathbf{y})\right) \in \mathbb{R}^{p} .
$$

From (3.17), it is easy to check that the function $\varphi_{2}$ defined in (3.18) satisfies (3.12). The point (3.10) is now proved.

d) Our new goal is to prove the following equality

$$
v(\mathbf{y})=-\eta u^{\prime}(\omega(\mathbf{y})) / H_{f}\left(\omega(\mathbf{y})+\eta^{2} \psi(\mathbf{y}),\right.
$$

with $\psi \in C^{k-2}\left(V, \mathbb{R}^{p}\right)$ and

$$
\left\|\partial^{\alpha} \psi(\mathbf{y})\right\| \ll \delta^{-3-|\alpha|}, \quad \text { for } \mathbf{y} \in V \text { and } 0 \leqslant|\alpha| \leqslant k-2,
$$

the constant implied above depending at most on $C, k$ and $p$.

Our starting point is the obvious equality

$$
\mathbf{0}=\mathbf{y}-\mathbf{y}=f_{\eta}^{\prime}\left(\omega_{\eta}(\mathbf{y})\right)-f^{\prime}(\omega(\mathbf{y}))
$$

which may be written as

$$
f^{\prime}(\omega(\mathbf{y})+v(\mathbf{y}))-f^{\prime}(\omega(\mathbf{y}))+\eta u^{\prime}(\omega(\mathbf{y})+v(\mathbf{y}))=\mathbf{0} .
$$

We apply (3.14) to the term $f^{\prime}(\omega(\mathbf{y})+v(\mathbf{y}))$ and (3.13) to the term $u^{\prime}(\omega(\mathbf{y})+$ $v(\mathbf{y}))$, so that $(3.21)$ becomes

$$
H_{f}(\omega(\mathbf{y})) v(\mathbf{y})=-\eta u^{\prime}(\omega(\mathbf{y}))-\eta^{2} \psi_{1}(\mathbf{y})
$$

with

$$
\begin{aligned}
\psi_{1}(\mathbf{y})= & \eta^{-2} \sum_{i, j=1}^{p} v_{i}(\mathbf{y}) v_{j}(\mathbf{y}) \int_{0}^{1}(1-t) \partial_{i} \partial_{j} f^{\prime}(\omega(\mathbf{y})+t v(\mathbf{y})) d t \\
& +\eta^{-1} \sum_{i=1}^{p} v_{i}(\mathbf{y}) \int_{0}^{1} \partial_{i} u^{\prime}(\omega(\mathbf{y})+t v(\mathbf{y})) d t .
\end{aligned}
$$
and

We apply (3.17) to the above formula to assert that we have $\psi_{1} \in C^{k-2}\left(V, \mathbb{R}^{p}\right)$

$$
\left\|\partial^{\alpha} \psi_{1}(\mathbf{y})\right\| \ll \delta^{-2-2|\alpha|}, \quad \text { for } \mathbf{y} \in V \text { and } 0 \leqslant|\alpha| \leqslant k-2 \text {. }
$$


We set

$$
\psi(\mathbf{y})=-\left(H_{f}(\omega(\mathbf{y}))^{-1} \psi_{1}(\mathbf{y})\right.
$$

and we deduce (3.19) from (3.22) and (3.23).

e) It remains to prove (3.11). As in part c), we write

$$
f_{\eta}^{*}(\mathbf{y})=f(\omega(\mathbf{y})+v(\mathbf{y}))+\eta u(\omega(\mathbf{y})+v(\mathbf{y}))-\langle\mathbf{y}, \omega(\mathbf{y})+v(\mathbf{y})\rangle .
$$

To the term $f(\omega(\mathbf{y})+v(\mathbf{y}))$, we apply Taylor's formula up to the third order, while the second order (cf. (3.14)) will be sufficient for the term $u(\omega(\mathbf{y})+v(\mathbf{y}))$. This yields

$$
\begin{aligned}
f_{\eta}^{*}(\mathbf{y})= & f^{*}(\mathbf{y})+\frac{1}{2}\left\langle H_{f}(\omega(\mathbf{y})) v(\mathbf{y}), v(\mathbf{y})\right\rangle \\
& +\eta u(\omega(\mathbf{y}))+\eta\left\langle u^{\prime}(\omega(\mathbf{y})), v(\mathbf{y})\right\rangle+\eta^{3} \varphi_{3}(\mathbf{y}),
\end{aligned}
$$

with

$$
\begin{aligned}
\varphi_{3}(\mathbf{y})= & \eta^{-3} \sum_{i, j, l=1}^{p} v_{i}(\mathbf{y}) v_{j}(\mathbf{y}) v_{l}(\mathbf{y}) \int_{0}^{1} \frac{(1-t)^{2}}{2} \partial_{i} \partial_{j} \partial_{l} f(\omega(\mathbf{y})+t v(\mathbf{y})) d t \\
& +\eta^{-2} \sum_{i, j=1}^{p} v_{i}(\mathbf{y}) v_{j}(\mathbf{y}) \int_{0}^{1}(1-t) \partial_{i} \partial_{j} u(\omega(\mathbf{y})+t v(\mathbf{y})) d t
\end{aligned}
$$

Applying (3.17), we see that $\varphi \in C^{k-3}\left(V, \mathbb{R}^{p}\right)$ and satisfies

$$
\left\|\partial^{\alpha} \varphi(\mathbf{y})\right\| \ll \delta^{-3-2|\alpha|}, \quad \text { for } \mathbf{y} \in V \text { and } 0 \leqslant|\alpha| \leqslant k-3 .
$$

Inserting this, as well as (3.19), in (3.25), we get (3.11). The proof of Proposition 3.2 is complete.

\subsection{Semi-monomial functions}

This section is left void.

\section{Oscillatory integrals}

In this section, we consider $p$-dimensional oscillatory integrals of the form

$$
\int \chi(\mathbf{x}) e(\tau(f(\mathbf{x})-\langle\mathbf{x}, \xi\rangle)) d \mathbf{x}
$$

where $\tau$ is a large positive number and $p$ is a fixed positive integer. The case without critical point reduces to a bound. If there exists a critical point, the injectivity of $f^{\prime}$ ensures that it is unique. As previously, the hypothesis

$$
\left|\operatorname{det} H_{f}(\mathbf{x})\right| \geqslant \delta>0
$$

is assumed for all $\mathbf{x}$ in the open set $\Omega$ where $f$ is defined, so that only nondegenerate critical points will be considered. As we have assumed that $\Omega$ is connected, the 
integer $\sigma\left(H_{f}(\mathbf{x})\right)$, defined in $(2.5)$, is constant when $\mathbf{x}$ stays in $\Omega$. That is why we may set

$$
\sigma=\sigma\left(H_{f}(\mathbf{x})\right), \quad \mathbf{x} \in \Omega .
$$

In our application to exponential sums, we shall use the asymptotics of such oscillatory integrals for an arbitrary large number of values of $\xi$ at the same time. Moreover, the support of $\chi$ is given by our initial problem and cannot be reduced. These considerations exclude the classical approaches more or less based on Morse's lemma, as may be found e.g. in Proposition 6 in page 344 of [13], where all parameters, except $\tau$, are fixed, and where $\chi$ must have a "sufficiently small" support.

Only Hörmander's approach, as in Theorem 7.7.5 of [3], which is more difficult, is sufficiently uniform to be applied here. For the convenience of the reader, or for a possible subsequent use, we have recalled it, restricted to the form we need, in Lemma 4.2 below.

The main complication in our paper comes from the fact that we want to include the additional possibility for $\delta$ to be abnormally small; this is dictated, among other applications, by the case where the phase function may be written as $f(\mathbf{x})=f_{0}(\mathbf{x}+\mathbf{h})-f_{0}(\mathbf{x})$ after a multidimensional $A$-process (cf [2]). The uniformity with respect to $\delta$ is not covered by Hörmander's result; an adaptation is possible, but difficult. Moreover, Hörmander's approach aims to give the complete asymptotic expansion of the oscillatory integral, while the main term plus an explicit error term is sufficient for our problem.

We have thus chosen to give a direct and new approach for the asymptotics of the stationary phase integral, in Theorem 4.1 below, based on spherical coordinates and a kind of Morse's lemma in two variables only. As a disadvantage, our error term is now $O\left(\tau^{-(p+1) / 2}\right)$ instead of the natural $O\left(\tau^{-p / 2-1}\right)$, as in Hörmander's Lemma 8; this is however sufficient for our needs. As an advantage, our proof may be adapted to an oscillatory integral whose critical point of the phase is also a strongly singular point of a particular type of the boundary of the domain of integration. This is the aim of Theorem 2 below, ${ }^{2}$ which is independent of the goal of this paper.

\subsection{Oscillatory integral without critical point}

Let $f \in C^{k}(\Omega, \mathbb{R})$, with $k=l+1 \geqslant 2$, satisfying

$$
\left|\partial^{\alpha} f(\mathbf{x})\right| \leqslant C \quad \text { for } 2 \leqslant|\alpha| \leqslant k \text { and } \mathbf{x} \in \Omega .
$$

Let $\chi \in C_{0}^{l}(\Omega, \mathbb{C})$. We set

$$
K=\text { support of } \chi, \quad|K|=\text { euclidean volume of } K, \quad \text { and } \quad L=f^{\prime}(K) .
$$

and, for any $\xi \in \mathbb{R}^{p}$

$$
d(\xi, L)=\inf _{\mathbf{y} \in L}\|\mathbf{y}-\xi\|
$$

\footnotetext{
${ }^{2}$ See Appendix.
} 
Let us give now an immediate consequence of Theorem 7.7.1 of [3].

Lemma 4.1. With the above notations, the bound

$$
\int \chi(\mathbf{x}) e(\tau(f(\mathbf{x})-\langle\mathbf{x}, \xi\rangle)) d \mathbf{x} \ll|K| \tau^{-l} \sum_{|\alpha| \leqslant l} \sup _{\mathbf{x}}\left|\partial^{\alpha} \chi(\mathbf{x})\right| d(\xi, L)^{|\alpha|-2 l}
$$

holds for any $\tau \geqslant 1$ and any $\xi \in \mathbb{R}^{p}, \xi \notin L$. The above implied constant depends only on $C, p$ and $k$.

Proof. If we set $g(\mathbf{x})=f(\mathbf{x})-\langle\mathbf{x}, \xi\rangle$, then formula $(7.7 .1)^{\prime}$ of [3] applied to $g$ implies (4.4); for this, we have just to note the inequality $\left\|g^{\prime}(\mathbf{x})\right\| \geqslant d(\xi, L)$. However, in our simpler case, the idea of the proof may be recalled in a few lines; for the convenience of the reader, we are going to prove (4.4) for $l=1$, the general situation being obtained by repeating the process. We write

$$
\chi(\mathbf{x}) e(\tau(f(\mathbf{x})-\langle\mathbf{x}, \xi\rangle))=\chi(\mathbf{x}) e(\tau g(\mathbf{x}))=\sum_{j=1}^{p} \frac{\chi(\mathbf{x}) \partial_{j} g(\mathbf{x})}{2 \pi i \tau\left\|g^{\prime}(\mathbf{x})\right\|^{2}} \frac{d}{d x_{j}}(e(\tau g(\mathbf{x})) .
$$

Integrating by parts thus yields

$$
\int \chi(\mathbf{x}) e(\tau(f(\mathbf{x})-\langle\mathbf{x}, \xi\rangle)) d \mathbf{x}=\frac{-1}{2 \pi i \tau} \sum_{j=1}^{p} \int\left(\frac{d}{d x_{j}}\left(\frac{\chi(\mathbf{x}) \partial_{j} g(\mathbf{x})}{\left\|g^{\prime}(\mathbf{x})\right\|^{2}}\right)\right) e(\tau g(\mathbf{x})) d \mathbf{x} .
$$

Now, expanding the derivative and giving rough bounds for each term give the desired result.

\subsection{Stationary phase integral without uniformity in $\delta$}

Let $f \in C^{k}(\Omega, \mathbb{R})$ be as in section 3.1, satisfying (3.1), (3.2) and (3.5). We define $\omega$, $U, f^{*}$ as in (3.3).

We are now ready to state a simplified version of Theorem 7.7.5 of [3].

Lemma 4.2. We suppose that $k=3 l+1$ for some positive integer $l$. Let $\chi \in$ $C_{0}^{2 l}(\Omega, \mathbb{C})$ which satisfies

$$
\left|\partial^{\beta} \chi(\mathbf{x})\right| \leqslant C_{1}, \quad \text { for } 0 \leqslant|\beta| \leqslant 2 l \text { and } \mathbf{x} \in \Omega .
$$

Let $\xi \in U$ such that

$$
C_{2}:=\sup _{\mathbf{x} \in \Omega}\|\mathbf{x}-\omega(\xi)\| /\left\|f^{\prime}(\mathbf{x})-\xi\right\|<+\infty .
$$

Then, for any real $\tau>1$, we have

$$
\int \chi(\mathbf{x}) e(\tau(f(\mathbf{x})-\langle\mathbf{x}, \xi\rangle)) d \mathbf{x}=\frac{e^{i \pi \sigma / 4} \chi(\omega(\xi)) e\left(\tau f^{*}(\xi)\right)}{\left|\operatorname{det} H_{f}(\omega(\xi))\right|^{1 / 2} \tau^{p / 2}}+O\left(\tau^{-p / 2-1}+\tau^{-l}\right) .
$$

The constant implied in the $O$ symbol above remains bounded when $C, C_{1}, C_{2}, p$, $k$, and the euclidean volume of the support of $\chi$ are uniformly bounded. 
Remark. With our hypotheses, we have a bound on $C_{2}$ in terms of $C$ and $p$, provided that we restrict slightly the open set $\Omega$. Indeed, it follows at once from Lemma A1 of [1], which is a uniform version of the inverse function theorem, that we have the precise lower bound:

Lemma 4.3. Let $f \in C^{3}(\Omega, \mathbb{R})$ a function which satisfies the properties:

$$
\begin{cases}\left|\partial^{\alpha} f(\mathbf{x})\right| \leqslant C, & \text { for } x \in \Omega \text { and } 2 \leqslant|\alpha| \leqslant 3 \\ \left|\operatorname{det} H_{f}(\mathbf{x})\right| \geqslant \delta, & \text { for } \mathbf{x} \in \Omega, \\ f^{\prime}: \Omega \rightarrow \mathbb{R}^{p} & \text { is injective. }\end{cases}
$$

We set

$$
\kappa=\frac{\delta}{2 p^{2} p ! C^{p}} .
$$

Then, for $\|\mathbf{h}\|<\kappa$ and $B(\mathbf{x}, \kappa) \subset \Omega$, we have

$$
\left\|f^{\prime}(\mathbf{x}+\mathbf{h})-f^{\prime}(\mathbf{x})\right\| \geqslant \frac{\delta}{4 p ! C^{p-1}}\|\mathbf{h}\|
$$

\subsection{Stationary phase integral with uniformity in $\delta$}

Let $f \in C^{k}(\Omega, \mathbb{R})$ be as in section 3.1, satisfying (3.1), (3.2) and (4.2). We define $\omega, U, f^{*}$ as in (3.3), and we keep the notations (4.1) and (4.3). Let $\chi \in C^{k}(\Omega, \mathbb{C})$ which satisfies

$$
\left|\partial^{\beta} \chi(\mathbf{x})\right| \leqslant C_{1}, \quad \text { for } 0 \leqslant|\beta| \leqslant k \text { and } \mathbf{x} \in \Omega .
$$

Let $\xi \in U$, and let us set

$$
\kappa=d\left(\omega(\xi), \Omega^{c}\right)
$$

Theorem 4.1. With the above notations and hypotheses, we assume that $k \geqslant p+5$.

We then have

$$
\begin{aligned}
\int \chi(\mathbf{x}) e(\tau(f(\mathbf{x})-\langle\mathbf{x}, \xi\rangle)) d \mathbf{x}= & \frac{e^{i \pi \sigma / 4} \chi(\omega(\xi)) e\left(\tau f^{*}(\xi)\right)}{\left|\operatorname{det} H_{f}(\omega(\xi))\right|^{1 / 2} \tau^{p / 2}} \\
& +O\left(C_{1} \frac{\delta^{-1 / 2} \kappa^{-1}+\delta^{-2}}{\tau^{(p+1) / 2}}\right) \\
& +O\left(\frac{C_{1}|K|\left(\kappa^{-4(k-1)}+\delta^{-6(k-1)}\right)}{\tau^{k-1}}\right)
\end{aligned}
$$

the implied constants depending at most on $C$ and $k$.

Proof. We split the proof into several steps.

All implied constants below depend at most on $C, p$ and $k$. 
a) Preliminaries. Up to a translation, we may suppose that $\omega(\xi)=\mathbf{0}$; this does not change the problem, except that we have now to assume that $f(\mathbf{0})=f^{*}(\xi)$. Let $A$ denote the matrix $H_{f}(\mathbf{0}) \in M_{p}(\mathbb{R})$ and let $\lambda_{1}, \ldots, \lambda_{m},-\mu_{1}, \ldots,-\mu_{n}$ be its eigenvalues with multiplicities. Here, we have $\lambda_{j}>0$ and $\mu_{j}>0$ for all $j$, $m+n=p$ and $\sigma=\sigma(A)=m-n$.

As in Lemma 2.1, we fix an orthogonal matrix $P$ such that

$$
P^{-1} A P=\left(\begin{array}{cccccc}
\lambda_{1} & \cdots & 0 & 0 & \cdots & 0 \\
\vdots & \ddots & \vdots & \vdots & \cdots & \vdots \\
0 & \cdots & \lambda_{m} & 0 & \cdots & 0 \\
0 & \cdots & 0 & -\mu_{1} & \cdots & 0 \\
\vdots & \cdots & \vdots & \vdots & \ddots & \vdots \\
0 & \cdots & 0 & 0 & \cdots & -\mu_{n}
\end{array}\right)
$$

Next, being given a small enough positive real number $\varepsilon$, to be determined later, we construct a function $\varrho \in C_{0}^{\infty}\left(\mathbb{R}^{p}, \mathbb{R}\right)$ such that

$$
\begin{aligned}
\varrho(\mathbf{x}) & =1 & & \text { for }\|\mathbf{x}\| \leqslant \varepsilon, \\
\varrho(\mathbf{x}) & =0 & & \text { for }\|\mathbf{x}\| \geqslant 2 \varepsilon ; \\
\left|\partial^{\alpha} \varrho(\mathbf{x})\right| & \ll \varepsilon^{-|\alpha|}, & & \text { for } \varepsilon \leqslant\|\mathbf{x}\| \leqslant 2 \varepsilon \text { and }|\alpha| \leqslant k,
\end{aligned}
$$

the above implied constant depending at most on $k$. We then set

$$
\chi_{1}(\mathbf{x})=\chi(\mathbf{x}) \varrho(\mathbf{x}) \quad \text { and } \quad \chi_{2}(\mathbf{x})=\chi(\mathbf{x})(1-\varrho(\mathbf{x}))
$$

and

$$
I_{\nu}=\int \chi_{\nu}(\mathbf{x}) e(\tau(f(\mathbf{x})-\langle\mathbf{x}, \xi\rangle)) d \mathbf{x}=\int \chi_{\nu}(\mathbf{x}) e(\tau g(\mathbf{x})) d \mathbf{x}, \quad \nu=1,2
$$

with $g(\mathbf{x})=f(\mathbf{x})-\langle\mathbf{x}, \xi\rangle$, so that our integral is equal to $I_{1}+I_{2}$. We shall study separately $I_{1}$ and $I_{2}$.

b) A first condition on $\varepsilon$. We want to determine a condition on $\varepsilon$ for which a suitable bound for $I_{2}$ will become possible. In order to apply Lemma 4.1, we need an effective version of the inverse function theorem. This is the aim of Lemma A.1 of [1]. More precisely, we set

$$
\varepsilon_{0}=\min \left\{\kappa, \frac{\delta}{2 p^{2} p ! C^{p}}\right\} .
$$

For $\varepsilon \leqslant \varepsilon_{0}$, say $\varepsilon=\vartheta \varepsilon_{0}$ for some $0<\vartheta \leqslant 1$, then Lemma A.1 of [1] asserts that the following inclusion holds:

$$
f^{\prime}(B(\mathbf{0}, \varepsilon)) \supset B\left(\xi, \frac{\vartheta \delta \varepsilon}{4 p ! C^{p-1}}\right),
$$

where we have set, as usual, $B(\mathbf{a}, \varepsilon)=\left\{\mathbf{x} \in \mathbb{R}^{p} \mid\|\mathbf{x}-\mathbf{a}\|<\varepsilon\right\}$. In other words, let $K_{2}$ denote the support of $\chi_{2}$ and let $L_{2}=f^{\prime}\left(K_{2}\right)$. By the injectivity of $f^{\prime}$, any 
$\mathbf{y} \in B\left(\xi, \frac{\vartheta \delta \varepsilon}{4 p ! C^{p-1}}\right)$, being the image of an $\mathbf{x} \in \Omega$ with $\mathbf{x} \notin K_{2}$, cannot be in $L_{2}$. We then have

$$
\varepsilon \leqslant \varepsilon_{0} \Longrightarrow d\left(\xi, L_{2}\right) \geqslant C_{2} \varepsilon^{2},
$$

for some positive $C_{2}$ which depends only on $C$ and $p$. An application of Lemma 4.1 yields

$$
I_{2} \ll C_{1}|K| \frac{\varepsilon^{-4(k-1)}}{\tau^{k-1}},
$$

providing that $\varepsilon \leqslant \varepsilon_{0}$.

c) The case $m=0$ or $n=0$. We are now interested in the asymptotic behavior of $I_{1}$; the simpler case $m=0$ or $n=0$ must be studied separately. We suppose, for instance, that $n=0$, and thus $m=p$. We recall the definition $I_{1}=\int \chi_{1}(\mathbf{x}) e(\tau g(\mathbf{x})) d \mathbf{x}$.

By Taylor's formula, we have

$$
\begin{aligned}
g(\mathbf{x})= & g(\mathbf{0})+\frac{1}{2}\langle A \mathbf{x}, \mathbf{x}\rangle+\psi(\mathbf{x})=f^{*}(\xi)+\frac{1}{2}\langle A \mathbf{x}, \mathbf{x}\rangle \\
& +\sum_{i, j, q=1}^{p} x_{i} x_{j} x_{q} \int_{0}^{1} \frac{(1-t)^{2}}{2} \partial_{i} \partial_{j} \partial_{q} f(t \mathbf{x}) d t
\end{aligned}
$$

providing that the segment $[\mathbf{0}, \mathbf{x}]$ is contained in $\Omega$, and this will be the case for $\|\mathbf{x}\|<\kappa$.

We want to apply the change of variables $\mathbf{x}=P \mathbf{y}$. We note that the orthogonality of $A$ and formula (4.11) yield

$$
\langle A \mathbf{x}, \mathbf{x}\rangle=\langle A P \mathbf{y}, P \mathbf{y}\rangle=\left\langle P^{-1} A P \mathbf{y}, \mathbf{y}\right\rangle=\sum_{j=1}^{p} \lambda_{j} y_{j}^{2}
$$

so that we get

$$
I_{1}=e\left(\tau f^{*}(\xi)\right) \int \chi_{1}(P \mathbf{y}) e\left(\frac{\tau}{2} \sum_{i=1}^{m} \lambda_{i} y_{i}^{2}+\tau \psi(P \mathbf{y})\right) d \mathbf{y} .
$$

Now, we set

$$
u_{1}=\sqrt{\lambda_{1}} y_{1}, \ldots, u_{p}=\sqrt{\lambda_{p}} y_{p} .
$$

This new change of variables in (4.19) yields

$$
I_{1}=\frac{e\left(\tau f^{*}(\xi)\right)}{\left|\operatorname{det} H_{f}(\omega(\xi))\right|^{1 / 2}} \int \chi_{0}(\mathbf{u}) e\left(\frac{\tau}{2}\|\mathbf{u}\|^{2}+\tau \psi_{0}(\mathbf{u})\right) d \mathbf{u},
$$

where the meaning of the notations $\chi_{0}$ and $\psi_{0}$ introduced above is obvious. In order to apply spherical coordinates, we set

$$
S^{p-1}=\left\{\mathbf{u} \in \mathbb{R}^{p} \mid\|\mathbf{u}\|=1\right\} \quad \text { and } \quad d S_{p-1}=\text { the surface measure on } S^{p-1}
$$


thus (4.20) becomes

$$
I_{1}=\frac{e\left(\tau f^{*}(\xi)\right)}{\left|\operatorname{det} H_{f}(\omega(\xi))\right|^{1 / 2}} \int_{S^{p-1}}\left(\int_{0}^{\infty} \chi_{0}(r \mathbf{u}) r^{p-1} e\left(\tau r^{2} / 2+\tau \psi_{0}(r \mathbf{u})\right) d r\right) d S_{p-1}(\mathbf{u}) .
$$

For $\mathbf{u}$ fixed in $S^{p-1}$ let $\Phi(\mathbf{u})$ denote the inner integral in the above formula, that is

$$
\Phi(\mathbf{u})=\int_{0}^{\infty} \chi_{0}(r \mathbf{u}) r^{p-1} e\left(\tau r^{2} / 2+\tau \psi_{0}(r \mathbf{u})\right) d r .
$$

In order to apply Lemma 2.5 , we want to justify the change of variables

$$
x=r \sqrt{1+2 \psi_{0}(r \mathbf{u}) r^{-2}}
$$

with the aim of transforming the integral (4.22) into

$$
\Phi(\mathbf{u})=\int_{0}^{\infty} \chi_{\mathbf{u}}(x) x^{p-1} e\left(\tau x^{2} / 2\right) d x
$$

The smaller eigenvalue of $A$ satisfies

$$
\lambda:=\min \left\{\lambda_{1}, \ldots, \lambda_{p}\right\} \gg \delta
$$

for some suitable positive implied constant that depends at most on $C$ and $p$. Indeed, we have $\lambda_{1} \ldots \lambda_{p}=\operatorname{det} A \geqslant \delta$, while each eigenvalue has an upper bound in terms of $C$ and $p$.

Now, we recall the definition of $\psi_{0}(r \mathbf{u})$. We set

$$
\widetilde{\mathbf{u}}=\left(\frac{u_{1}}{\sqrt{\lambda_{1}}}, \ldots, \frac{u_{p}}{\sqrt{\lambda_{p}}}\right)
$$

and we introduce the vectorial notation

$$
P \mathbf{y}=\left(P_{1}(\mathbf{y}), \ldots, P_{p}(\mathbf{y})\right) \in \mathbb{R}^{p},
$$

so that we may write

$$
\psi_{0}(r \mathbf{u})=r^{3} \sum_{i, j, q=1}^{p} P_{i}(\widetilde{\mathbf{u}}) P_{j}(\widetilde{\mathbf{u}}) P_{q}(\widetilde{\mathbf{u}}) \int_{0}^{1} \frac{(1-t)}{2} \partial_{i} \partial_{j} \partial_{q} f(\operatorname{tr} P(\widetilde{\mathbf{u}})) d t
$$

according to (4.17) and to the fact that we have set successively

$$
\mathbf{x}=P \mathbf{y}, \quad \mathbf{y}=r \widetilde{\mathbf{u}}, \quad \text { with } \quad\|\mathbf{u}\|=1, \text { and } \psi(\mathbf{x})=\psi(r P(\widetilde{\mathbf{u}}))=\psi_{0}(r \mathbf{u}) .
$$

In particular, we have the bounds, for $\mathbf{u} \in S^{p-1}$, and $0 \leqslant r<\kappa$

$$
r^{-2} \psi_{0}(r \mathbf{u}) \ll r \lambda^{-3 / 2}
$$


and

$$
\frac{d^{j}}{d r^{j}}\left(r^{-2} \psi_{0}(r \mathbf{u})\right) \ll \lambda^{-1-j / 2}, \quad \text { for } 1 \leqslant j \leqslant k-3 \text { and } r \ll \lambda^{-1 / 2} .
$$

The change of variables (4.23) will be justified if we ensure that $\left|2 r^{-2} \psi_{0}(r \mathbf{u})\right| \leqslant$ $1 / 2$, that is, if $r \ll \lambda^{3 / 2}$, or $r \ll \delta^{3 / 2}$, according to (4.25), for a sufficiently small implied constant. Recalling that $\chi_{0}(r \mathbf{u})=\chi(r P(\widetilde{\mathbf{u}})) \varrho(r P(\widetilde{\mathbf{u}}))$, we see that

$$
r\|P(\widetilde{\mathbf{u}})\| \geqslant 2 \varepsilon \Longrightarrow \chi_{0}(r \mathbf{u})=0 .
$$

But

$$
\|P(\widetilde{\mathbf{u}})\|=\|\widetilde{\mathbf{u}}\|=\left(\sum_{i=1}^{p} \frac{u_{i}^{2}}{\lambda_{i}}\right)^{1 / 2} \gg\|\mathbf{u}\|=1,
$$

so that there exists a constant $\eta>0$, small enough, depending at most on $C$ and $k$, such that

$$
r \leqslant \eta \delta^{3 / 2} \Longrightarrow\left|2 r^{-2} \psi_{0}(r \mathbf{u})\right| \leqslant 1 / 2 \quad \text { and } \quad r \geqslant \varepsilon / \eta \Longrightarrow \chi_{0}(r \mathbf{u})=0 .
$$

We fix $\eta$ as above and we set

$$
\varepsilon=\min \left(\eta^{2} \delta^{3 / 2}, \varepsilon_{0}\right) .
$$

For this choice of $\varepsilon$, then (4.15) holds and we have both

$$
\Phi(\mathbf{u})=\int_{0}^{\varepsilon / \eta} \chi_{0}(r \mathbf{u}) r^{p-1} e\left(\frac{1}{2} \tau r^{2}\left(1+2 r^{-2} \psi_{0}(r \mathbf{u})\right)\right) d r
$$

and

$$
\left|2 r^{-2} \psi_{0}(r \mathbf{u})\right| \leqslant 1 / 2 \quad \text { for } 0<r \leqslant \varepsilon / \eta,
$$

so that the change of variables (4.23) is now justified for $r \in[0, \varepsilon / \eta]$. We thus set

$$
x=r \sqrt{1+2 r^{-2} \psi(r \mathbf{u})} \Longleftrightarrow r=x(1+\varphi(x)),
$$

and that defines a function $\varphi:[0, c] \rightarrow \mathbb{R}$, for some $c$ of the same size as $\varepsilon$, such that

$$
\varphi(0)=0, \quad|\varphi(x)| \leqslant 1 / 2 \quad \text { and } \quad \varphi^{(j)}(x) \ll \delta^{-3 j / 2}, \quad \text { for } 1 \leqslant j \leqslant k-3,
$$

according to (4.30). Now, the function $\chi_{\mathbf{u}}:[0,+\infty[\rightarrow \mathbb{R}$, defined in (4.24), may be written as

$$
\chi_{\mathbf{u}}(x)=\chi_{0}(x(1+\varphi(x)) \mathbf{u})(1+\varphi(x))^{p-1}\left(1+\varphi(x)+x \varphi^{\prime}(x)\right)
$$

for $x \in[0, c]$, and $\chi_{\mathbf{u}}(x)=0$ for $x \geqslant c$. 
We may now apply Lemma 2.5 to the integral

$$
\Phi(\mathbf{u})=\int_{0}^{\infty} \chi_{\mathbf{u}}(x) x^{p-1} e\left(\tau x^{2} / 2\right) d x
$$

for some $c \gg \min \left(\kappa, \delta^{3 / 2}\right)$ and some $Z \ll \delta^{-3 / 2}$ and we get the estimation

$$
\Phi(\mathbf{u})=\frac{\Gamma(p / 2)}{2 \pi^{p / 2}} \chi(\mathbf{0}) e^{p i \pi / 4} \tau^{-p / 2}+O\left(C_{1} \frac{\kappa^{-1}+\delta^{-3 / 2}}{\tau^{(p+1) / 2}}\right) .
$$

We insert this in (4.21); recalling that the $(p-1)$-dimensional area of $S^{p-1}$ is equal to $2 \pi^{p / 2} \Gamma(p / 2)^{-1}$, we obtain

$$
I_{1}=\frac{\chi(\mathbf{0}) e^{p i \pi / 4} e\left(\tau f^{*}(\xi)\right)}{\left|\operatorname{det} H_{f}(\omega(\xi))\right|^{1 / 2}} \tau^{-p / 2}+O\left(C_{1} \frac{\delta^{-1 / 2} \kappa^{-1}+\delta^{-2}}{\tau^{(p+1) / 2}}\right) .
$$

Combining this with (4.15), the choice of $\varepsilon$ being as in (4.32), we obtain exactly (4.10) in the case $n=0$.

d) The case $m \geqslant 1$ and $n \geqslant 1$. We are now studying the asymptotic behavior of $I_{1}$ in the case where both $m$ and $n$ are nonzero. The above method may be used again with some modifications, the main ones being contained in Lemma 2.4 and Lemma 2.6. We give now the details.

The first modification occurs in (4.17), where we have to set

$$
\langle A \mathbf{x}, \mathbf{x}\rangle=\langle A P \mathbf{y}, P \mathbf{y}\rangle=\left\langle P^{-1} A P \mathbf{y}, \mathbf{y}\right\rangle=\sum_{i=1}^{m} \lambda_{i} y_{i}^{2}-\sum_{i=1}^{n} \mu_{m+i} y_{m+i}^{2} .
$$

From this, we deduce

$$
I_{1}=e\left(\tau f^{*}(\xi)\right) \int \chi_{1}(P \mathbf{y}) e\left(\frac{\tau}{2}\left(\sum_{i=1}^{m} \lambda_{i} y_{i}^{2}-\sum_{i=1}^{n} \mu_{n+i} y_{n+i}^{2}\right)+\tau \psi(P \mathbf{y})\right) d \mathbf{y}
$$

Introducing the second change of variables

$$
u_{1}=\sqrt{\lambda_{1}} y_{1}, \ldots, u_{m}=\sqrt{\lambda_{m}} y_{m} \quad \text { and } \quad v_{1}=\sqrt{\mu_{1}} y_{m+1}, \ldots, v_{n}=\sqrt{\mu_{n}} y_{m+n},
$$

we get

$$
I_{1}=\frac{e\left(\tau f^{*}(\xi)\right)}{\left|\operatorname{det} H_{f}(\omega(\xi))\right|^{1 / 2}} \int_{\mathbb{R}^{m}} \int_{\mathbb{R}^{n}} \chi_{0}(\mathbf{u}, \mathbf{v}) e\left(\frac{\tau}{2}\left(\|\mathbf{u}\|_{m}^{2}-\|\mathbf{v}\|_{n}^{2}\right)+\tau \psi_{0}(\mathbf{u}, \mathbf{v})\right) d \mathbf{u} d \mathbf{v}
$$

where $\|\mathbf{u}\|_{m}$ and $\|\mathbf{v}\|_{n}$ denote the euclidean norm of $\mathbf{u}$ in $\mathbb{R}^{m}$ and of $\mathbf{v}$ in $\mathbb{R}^{n}$, and where $\chi_{0}(\mathbf{u}, \mathbf{v})$ and $\chi_{0}(\mathbf{u}, \mathbf{v})$ have an obvious meaning.

We are now ready to apply the third change of variables, using spherical coordinates both for $\mathbf{u}$ and $\mathbf{v}$ :

$$
I_{1}=\frac{e\left(\tau f^{*}(\xi)\right)}{\left|\operatorname{det} H_{f}(\omega(\xi))\right|^{1 / 2}} \int_{S^{m-1}} \int_{S^{n-1}} \Phi(\mathbf{u}, \mathbf{v}) d S_{m-1}(\mathbf{u}) d S_{n-1}(\mathbf{v}),
$$


with

$$
\Phi(\mathbf{u}, \mathbf{v})=\int_{0}^{+\infty} \int_{0}^{+\infty} r^{m-1} s^{n-1} \chi_{0}(r \mathbf{u}, s \mathbf{v}) e\left(\tau\left(\frac{r^{2}}{2}-\frac{s^{2}}{2}+\psi_{0}(r \mathbf{u}, s \mathbf{v})\right)\right) d r d s
$$

From now on, we work only with this last double integral, and thus, we may fix $\mathbf{u} \in S^{m-1}$ and $\mathbf{v} \in S^{n-1}$. Our first goal is to give an appropriate form for $\psi_{0}(r \mathbf{u}, s \mathbf{v})$. We set

$$
\widetilde{\mathbf{u}}=\left(\frac{u_{1}}{\sqrt{\lambda_{1}}}, \ldots, \frac{u_{m}}{\sqrt{\lambda_{m}}}\right) \quad \text { and } \quad \widetilde{\mathbf{v}}=\left(\frac{v_{1}}{\sqrt{\mu_{1}}}, \ldots, \frac{v_{n}}{\sqrt{\mu_{n}}}\right) .
$$

According to (4.16), and with the notation (4.45), we have

$$
\begin{aligned}
\psi_{0}(r \mathbf{u}, s \mathbf{v})= & \sum_{i, j, q=1}^{p} P_{i}(r \widetilde{\mathbf{u}}, s \widetilde{\mathbf{v}}) P_{j}(r \widetilde{\mathbf{u}}, s \widetilde{\mathbf{v}}) P_{q}(r \widetilde{\mathbf{u}}, s \widetilde{\mathbf{v}}) \\
& \times \int_{0}^{1} \frac{(1-t)}{2} \partial_{i} \partial_{j} \partial_{q} f(t P(r \widetilde{\mathbf{u}}, s \widetilde{\mathbf{v}})) d t
\end{aligned}
$$

We write each of the linear forms $P_{i}, P_{j}$ and $P_{q}$ as a sum of $p$ terms containing $r$ or $s$ so that we have

$$
P_{i}(r \widetilde{\mathbf{u}}, s \widetilde{\mathbf{v}}) P_{j}(r \widetilde{\mathbf{u}}, s \widetilde{\mathbf{v}}) P_{q}(r \widetilde{\mathbf{u}}, s \widetilde{\mathbf{v}})=a_{1} r^{3}+a_{2} r^{2} s+a_{3} r s^{2}+a_{4} s^{3},
$$

where the $a_{\nu}$, for $\nu=1, \ldots, 4$, are coefficients depending only on $P, \widetilde{\mathbf{u}}$ and $\widetilde{\mathbf{v}}$; according to the following analogue of (4.26)

$$
\min \left\{\lambda_{1}, \ldots, \lambda_{m}, \mu_{1}, \ldots, \mu_{n}\right\} \gg \delta .
$$

we see that their size is $O\left(\delta^{-3 / 2}\right)$. Among the terms above, we group together those containing $r^{2}$ on the one side and those containing $s^{2}$ on the other side. Finally, we obtain the following form:

$$
\psi_{0}(r \mathbf{u}, s \mathbf{v})=r^{2} \varphi_{1}(r, s)+s^{2} \varphi_{2}(r, s), \quad \text { with } \varphi_{\nu}(0,0)=0 \text { for } \nu=1,2 .
$$

Moreover, using again (4.47), we deduce

$$
\partial^{\alpha} \varphi_{\nu}(r, s) \ll \delta^{-1-|\alpha| / 2} \quad \text { for } r+s \ll \delta^{-1 / 2} \text { and } 1 \leqslant|\alpha| \leqslant k-3,
$$

which can be used in the weaker form

$$
\left|\partial^{\alpha} \varphi_{\nu}(r, s)\right| \leqslant\left(C_{2} \delta^{-3 / 2}\right)^{|\alpha|} \quad \text { for }(r, s) \in\left[0, \delta^{-1 / 2} / C_{2}\right]^{2} \text { and } 1 \leqslant|\alpha| \leqslant k-3 \text {, }
$$

where $C_{2}$ is a suitable constant that depends at most on $C$ and $k$. We fix $C_{2}$ and we set $Z=C_{2} \delta^{-3 / 2}$.

We are now going to determine a possible choice for $\varepsilon$. We have the identity

$$
\chi_{0}(r \mathbf{u}, s \mathbf{v})=\chi_{0}(P(r \widetilde{\mathbf{u}}, s \widetilde{\mathbf{v}}))=0 \quad \text { for } \quad\|P(r \widetilde{\mathbf{u}}, s \widetilde{\mathbf{v}})\| \geqslant 2 \varepsilon .
$$


The orthogonality of $P$ and (4.45) show that

$$
\|P(r \widetilde{\mathbf{u}}, s \widetilde{\mathbf{v}})\|=\|(r \widetilde{\mathbf{u}}, s \widetilde{\mathbf{v}})\| \gg \max (r, s) .
$$

We have thus proved the existence of a constant $C_{3}$, that depends at most on $C$ and $k$, such that

$$
\max (r, s) \geqslant C_{3} \varepsilon \Longrightarrow \chi_{0}(r \mathbf{u}, s \mathbf{v})=0 .
$$

In order to justify the change of variables

$$
\left\{\begin{array} { l } 
{ x = r \sqrt { 1 + 2 \varphi _ { 1 } ( r , s ) } } \\
{ y = s \sqrt { 1 - 2 \varphi _ { 2 } ( r , s ) } }
\end{array} \Longleftrightarrow \left\{\begin{array}{l}
r=x\left(1+w_{1}(x, y)\right) \\
s=y\left(1+w_{2}(x, y)\right)
\end{array}\right.\right.
$$

as in Lemma 2.4, we choose $\varepsilon \ll Z^{-1}$, for a sufficiently small implied positive constant. Our final choice for $\varepsilon$ will be

$$
\varepsilon=\min \left(\varepsilon_{0}, \frac{\delta^{3 / 2}}{C_{4}}\right)
$$

for a sufficiently large constant $C_{4}$, and where $\varepsilon_{0}$ has been defined in (4.14). We have thus

$$
\Phi(\mathbf{u}, \mathbf{v})=\int_{0}^{c} \int_{0}^{c} r^{m-1} s^{n-1} \chi_{0}(r \mathbf{u}, s \mathbf{v}) e\left(\tau\left(\frac{r^{2}}{2}-\frac{s^{2}}{2}+\psi_{0}(r \mathbf{u}, s \mathbf{v})\right)\right) d r d s
$$

for some $c \gg \min \left(\kappa, \delta^{3 / 2}\right)$ and $c \ll Z^{-1}$. Applying Lemma 2.4, we get

$$
\Phi(\mathbf{u}, \mathbf{v})=\int_{0}^{+\infty} \int_{0}^{+\infty} x^{m-1} y^{n-1} \chi_{\mathbf{u}, \mathbf{v}}(x, y) e\left(\tau \frac{x^{2}}{2}-\tau \frac{y^{2}}{2}\right) d x d y
$$

with

$$
\chi_{\mathbf{u}, \mathbf{v}}(0,0)=\chi(\mathbf{0})=\chi(\omega(\xi)), \quad \chi_{\mathbf{u}, \mathbf{v}}(x, y)=0 \quad \text { for } \quad \max (x, y) \geqslant c,
$$

and

$$
\partial^{\alpha} \chi_{\mathbf{u}, \mathbf{v}}(x, y) \ll C_{1} \delta^{-3|\alpha| / 2}, \quad \text { for } 0 \leqslant|\alpha| \leqslant k-3 .
$$

For proving this last assertion, we note the formula

$$
\begin{aligned}
\chi_{\mathbf{u}, \mathbf{v}}(x, y)= & \chi_{1}(P(r(x, y) \widetilde{\mathbf{u}}, s(x, y) \widetilde{\mathbf{v}})) \\
& \times\left(1+w_{1}(x, y)\right)^{m-1}\left(1+w_{2}(x, y)\right)^{n-1} \times J(x, y)
\end{aligned}
$$

where $J(x, y)$ denotes the Jacobian of the change of variables, and is equal to $\left(1+x \partial_{1} w_{1}(x, y)+w_{1}(x, y)\right)\left(1+y \partial_{2} w_{2}(x, y)+w_{2}(x, y)\right)-x y \partial_{2} w_{1}(x, y) \partial_{1} w_{2}(x, y)$ and where the functions $w_{1}(x, y)$ and $w_{2}(x, y)$ are defined in (4.52). Thus, (4.57) may be deduced from (2.13) and (2.18), with $Z=C_{2} \delta^{-3 / 2}$. 
We want to apply Lemma 2.6. This requires that $w_{\nu} \in C^{k-1}\left(\left[0,+\infty\left[^{2}\right)\right.\right.$, for $\nu=1,2$, while, with the notations of Lemma 2.4, we know only that $w_{\nu} \in$ $C^{k-1}(W)$ by Lemma 2.4. However, if we take $C_{4}$ large enough in (4.53), the support of $\chi_{\mathbf{u}, \mathbf{v}}$ will be contained in $[0, c / 2]^{2} \subset W$ for our choice of $c$, so that Lemma 2.3 may be applied to $w_{\nu}$ on this domain. We note also that, if hypothesis (2.34) is not satisfied, then (4.10) is obvious, the second error term being greater than the trivial bound of the integral. These remarks make it possible to apply Lemma 2.6.

Thus, we have

$$
\Phi(\mathbf{u}, \mathbf{v})=\frac{\Gamma(m / 2) \Gamma(n / 2)}{4(2 \pi)^{p / 2}} \chi(\omega(\xi)) e^{i \sigma \pi / 4} \tau^{-p / 2}+O\left(\frac{\kappa^{-1}+\delta^{3 / 2}}{\tau^{(p+1) / 2}}\right),
$$

providing that $\tau \gg \kappa^{-2}+\delta^{-3}$, with a sufficiently large implied constant, as required in (2.34). We report this last equality in (4.43) to obtain the value of $I_{1}$, and according to (4.15), we get finally (4.10). The theorem is proved.

\section{The multidimensional $B$-process}

\subsection{Hypotheses and notations}

For the convenience of the reader, we recall here all the notations and hypotheses used in the statement of the following two theorems.

Let $\Omega$ be a connected open set of $\mathbb{R}^{p}$ and let $f \in C^{k}(\Omega, \mathbb{R})$ be the phase function, where $k \geqslant p+5$ is a fixed integer. Let also $\chi \in C_{0}^{k}(\Omega, \mathbb{C})$ be the amplitude of the exponential sum. Let furthermore $D$ be any compact of $\mathbb{R}^{p}$.

Let $T, M_{1}, \ldots, M_{p}$ be $p+1$ large real numbers (at least greater than 1 , say). We consider the two exponential sums

$$
S=\sum_{\mathbf{m} \in \mathbb{Z}^{p}} \chi\left(\frac{m_{1}}{M_{1}}, \ldots, \frac{m_{p}}{M_{p}}\right) e\left(T f\left(\frac{m_{1}}{M_{1}}, \ldots, \frac{m_{p}}{M_{p}}\right)\right)
$$

and

$$
S_{D}=\sum_{\substack{\mathbf{m} / \mathbf{M} \in D \\ \mathbf{m} \in \mathbb{Z}^{p}}} \chi\left(\frac{m_{1}}{M_{1}}, \ldots, \frac{m_{p}}{M_{p}}\right) e\left(T f\left(\frac{m_{1}}{M_{1}}, \ldots, \frac{m_{p}}{M_{p}}\right)\right) .
$$

Here and in the sequel we assume that $\chi$ vanishes outside the open set $\Omega$. We shall call $S$ the exponential sum without boundary, and the $S_{D}$ exponential sum with boundary.

The first basic hypothesis is

$$
\left|\operatorname{det} H_{f}(\mathbf{x})\right| \geqslant \delta, \quad \text { for } \mathbf{x} \in \Omega,
$$

for some $\delta(0<\delta \leqslant 1)$ which may be small. This implies that the signature of $H_{f}(\mathbf{x})$ is constant over $\Omega$, and equal to $\left(p^{+}, p^{-}\right)$, say, and we set

$$
\sigma=p^{+}-p^{-} \text {. }
$$


We suppose that we have

$$
\begin{array}{ll}
\left|\partial^{\alpha} f(\mathbf{x})\right| \leqslant C, & \text { for } 2 \leqslant|\alpha| \leqslant k \text { and } \mathbf{x} \in \Omega \\
\left|\partial^{\alpha} \chi(\mathbf{x})\right| \leqslant C, & \text { for } 0 \leqslant|\alpha| \leqslant k \text { and } \mathbf{x} \in \Omega,
\end{array}
$$

for some fixed constant $C \geqslant 2$, and that $\Omega$ is contained in a bounded hypercube, say

$$
\Omega \subset \mathbf{a}+[0,2 C]^{p},
$$

for some $\mathbf{a} \in \mathbb{R}^{p}$. We define $K$ as being the support of $\chi$ and we suppose that

$$
d\left(K, \Omega^{c}\right) \geqslant 1 /(3 C)
$$

Now, we recall the definition of $f^{*}$, already studied in detail in $\S 2$. Our second basic hypothesis is

$$
f^{\prime}: \Omega \rightarrow \mathbb{R}^{p} \quad \text { is injective. }
$$

We then set $U=f^{\prime}(\Omega)$ and we define $\omega: U \rightarrow \Omega$ as the inverse map of $f^{\prime}$. We may now introduce the new phase function $f^{*}: U \rightarrow \mathbb{R}$ as

$$
f^{*}(\mathbf{y})=f(\omega(\mathbf{y}))-\langle\mathbf{y}, \omega(\mathbf{y})\rangle
$$

whose properties are gathered in $\S 3$.

We set $N_{i}=T / M_{i}$, for $i=1, \ldots, p$, and we shall use the simplified notation

$$
\begin{aligned}
\frac{\mathbf{m}}{\mathbf{M}} & =\left(\frac{m_{1}}{M_{1}}, \ldots, \frac{m_{p}}{M_{p}}\right), \quad \mathbf{n} / \mathbf{N}=\left(\frac{n_{1}}{N_{1}}, \ldots, \frac{n_{p}}{N_{p}}\right), \\
\frac{\mathbf{n}+\vartheta}{\mathbf{N}} & =\left(\frac{n_{1}+\vartheta_{1}}{N_{1}}, \ldots, \frac{n_{p}+\vartheta_{p}}{N_{p}}\right) \in \mathbb{R}^{p} .
\end{aligned}
$$

\subsection{The $B$-process without boundary}

We introduce the quantity

$$
R_{0}=\frac{M_{1} \cdots M_{p}}{T^{p}} \sum_{\mathbf{n} / \mathbf{N} \in f^{\prime}(\Omega)} 1
$$

which occurs in the main error term and satisfies the inequality

$$
R_{0} \ll \frac{M_{1} \cdots M_{p}}{T^{p}} \prod_{i=1}^{p}\left(1+T / M_{i}\right) .
$$

In main applications, when $T$ is large enough (say $T \geqslant 2 M_{i}$ for $i=1, \ldots, p$ ), this term is $O(1)$; but in other cases, $R_{0}$ may be small (possibly zero) or large. 
Theorem 5.2. ${ }^{3}$ All the notations and hypotheses are those of section 5.1. We then have

$$
\begin{aligned}
S= & \frac{M_{1} \cdots M_{p}}{T^{p / 2}} e^{i \pi \sigma / 4} \sum_{\mathbf{n} \in \mathbb{Z}^{p}} \frac{\chi\left(\omega\left(\frac{\mathbf{n}}{\mathbf{N}}\right)\right)}{\left|\operatorname{det} H_{f}\left(\omega\left(\frac{\mathbf{n}}{\mathbf{N}}\right)\right)\right|^{1 / 2}} e\left(T f^{*}\left(\frac{\mathbf{n}}{\mathbf{N}}\right)\right) \\
& +O\left(R_{0} T^{(p-1) / 2} \delta^{-2}\right)+O\left(\frac{R_{0} T^{p}}{\left(T \delta^{6}\right)^{k-1}}\right) \\
& +O\left(\left(\prod_{i=1}^{p}\left(T+M_{i}\right)\right)\left(T \delta^{2}\right)^{-(k-1)}\right),
\end{aligned}
$$

the implied constants depending at most on $C$ and $k$.

\section{Remarks.}

1. The standard situation is obtained when $T / M_{i}$ is large and $T / M_{i}^{2}$ is small, for each $i=1, \ldots, p$, and when $\delta$ is not small. We suppose, in this remark, that these conditions hold. If we bound trivially the new exponential sum in (5.11), the main term is $O\left(T^{p / 2}\right)$, the error terms in (5.11) being smaller.

Before applying the $B$-process, a trivial bound of $S$ as defined in (5.1) gives $S=O\left(M_{1} \cdots M_{p}\right)$. We thus have obtained a saving of $\left(\prod_{i=1}^{p} T / M_{i}^{2}\right)^{1 / 2}$, that is, we save on each variable exactly the same quantity as with the onedimensional van der Corput's inequality (cf. Theorem 2.2. of [2]). In other words, in the standard case, we have

$$
S \ll T^{p / 2}=M_{1} \cdots M_{p}\left(\prod_{i=1}^{p} T / M_{i}^{2}\right)^{1 / 2} .
$$

This is the natural extension of the one-dimensional van der Corput's inequality when there is no boundary problem.

2. We still suppose that we are in the standard situation; the transformation (5.11), after a saving of $\left(\prod_{i=1}^{p} T / M_{i}^{2}\right)^{1 / 2}$, yields a new multiple exponential sum. A further saving may be expected. But the new phase function $T f^{*}$ is as large as the previous one, while the new variable $\mathbf{n}$ is much shorter. It is well known that only a weak new saving is expected, so that the error terms in (5.11) need not to be sharp, at least for the present time.

If necessary, one may improve our error term, using Hörmander's Lemma 8 instead of our Theorem 4.1. This should yield

$$
S=\frac{M_{1} \cdots M_{p}}{T^{p / 2}} e^{i \pi \sigma / 4} \sum_{\mathbf{n} \in \mathbb{Z}^{p}} \frac{\chi(\omega(\mathbf{n} / \mathbf{N}))}{\left|\operatorname{det} H_{f}(\omega(\mathbf{n} / \mathbf{N}))\right|^{1 / 2}} e\left(T f^{*}\left(\frac{\mathbf{n}}{\mathbf{N}}\right)\right)+O\left(T^{p / 2-1}\right) .
$$

A precise formulation should require some more work that will not be done here.

\footnotetext{
${ }^{3}$ Since a statement seems to be missing, we increased the counter by 1 in order to fit with Sargos' numbering.
} 
Proof of Theorem 5.2. We recall first that the Poisson summation formula

$$
\sum_{\mathbf{m} \in \mathbb{Z}^{p}} \varrho(\mathbf{m})=\sum_{\mathbf{n} \in \mathbb{Z}^{p}} \widehat{\varrho}(\mathbf{n})
$$

with $\widehat{\varrho}(\mathbf{y})=\int_{\mathbb{R}^{p}} \varrho(\mathbf{x}) e(-\langle\mathbf{x}, \mathbf{y}\rangle) d \mathbf{x}$ holds for any $\varrho \in C_{0}^{p+1}\left(\mathbb{R}^{p}, \mathbb{C}\right)$. Taking

$$
\varrho(\mathbf{m})=\chi\left(\frac{\mathbf{m}}{\mathbf{M}}\right) e\left(T f\left(\frac{\mathbf{m}}{\mathbf{M}}\right)\right),
$$

the sum $S$, defined in (5.1), becomes

$$
\begin{aligned}
S & =\sum_{\mathbf{n} \in \mathbb{Z}^{p}} \int_{\mathbb{R}^{p}} \chi\left(\frac{\mathbf{x}}{\mathbf{M}}\right) e\left(T f\left(\frac{\mathbf{x}}{\mathbf{M}}\right)-\langle\mathbf{x}, \mathbf{n}\rangle\right) d \mathbf{x} \\
& =M_{1} \cdots M_{p} \sum_{\mathbf{n} \in \mathbb{Z}^{p}} \int_{\mathbb{R}^{p}} \chi(\mathbf{y}) e\left(T\left(f(\mathbf{y})-\left\langle\mathbf{y}, \frac{\mathbf{n}}{\mathbf{N}}\right\rangle\right)\right) d \mathbf{y},
\end{aligned}
$$

with the change of variables $\mathbf{y}=\frac{\mathbf{x}}{\mathbf{M}}$,

$$
=M_{1} \cdots M_{p} \sum_{\mathbf{n} \in \mathbb{Z}^{p}} J(\mathbf{n}), \quad \text { say. }
$$

Now, we may apply the results of $\S 3$ to the last integral. By Lemma 4.1, we have

$$
J(\mathbf{n})=\frac{e^{i \pi \sigma / 4} \chi(\omega(\mathbf{n} / \mathbf{N})) e\left(T f^{*}(\mathbf{n} / \mathbf{N})\right)}{\left|\operatorname{det} H_{f}(\omega(\mathbf{n} / \mathbf{N}))\right|^{1 / 2} T^{p / 2}}+R_{1}(\mathbf{n}),
$$

with

$$
R_{1}(\mathbf{n}) \ll T^{-(k-1)} d\left(\frac{\mathbf{n}}{\mathbf{N}}, L\right)^{-(k-1)}\left(1+d\left(\frac{\mathbf{n}}{\mathbf{N}}, L\right)^{-(k-1)}\right),
$$

provided that $\mathbf{n} / \mathbf{N} \notin L=f^{\prime}(K)$. Indeed, recalling that $K$ is the support of $\chi$, as $\omega(\mathbf{n} / \mathbf{N}) \notin K$, we have $\chi(\omega(\mathbf{n} / \mathbf{N}))=0$. Thus (5.13) may be reduced to $J(\mathbf{n})=R_{1}(\mathbf{n})$, and this is a simple consequence of Lemma 4.1.

By Theorem 4.1, we have

$$
J(\mathbf{n})=\frac{e^{i \pi \sigma / 4} \chi(\omega(\mathbf{n} / \mathbf{N})) e\left(T f^{*}(\mathbf{n} / \mathbf{N})\right)}{\left|\operatorname{det} H_{f}(\omega(\mathbf{n} / \mathbf{N}))\right|^{1 / 2} T^{p / 2}}+R_{2}(\mathbf{n}),
$$

with

$$
R_{2}(\mathbf{n}) \ll \delta^{-2} T^{-(p+1) / 2}+\left(\delta^{6} T\right)^{-(k-1)},
$$

provided that

$$
d\left(\omega\left(\frac{\mathbf{n}}{\mathbf{N}}\right), \Omega^{c}\right) \gg 1
$$


We split the set of $\mathbf{n} \in \mathbb{Z}^{p}$, according to the fact that $d\left(\omega\left(\frac{\mathbf{n}}{\mathbf{N}}\right), \Omega^{c}\right)$ is $\leqslant(2 C)^{-1}$ or $>(2 C)^{-1}$. This yields

$$
\begin{aligned}
S= & M_{1} \cdots M_{p} e^{i \pi \sigma / 4} \sum_{\mathbf{n} \in \mathbb{Z}^{p}} \frac{\chi(\omega(\mathbf{n} / \mathbf{N})) e\left(T f^{*}(\mathbf{n} / \mathbf{N})\right)}{\left|\operatorname{det} H_{f}(\omega(\mathbf{n} / \mathbf{N}))\right|^{1 / 2} T^{p / 2}}+ \\
& +M_{1} \cdots M_{p}\left(\sum_{d\left(\omega(\mathbf{n} / \mathbf{N}), \Omega^{c}\right) \leqslant(2 C)^{-1}} R_{1}(\mathbf{n})+\sum_{d\left(\omega(\mathbf{n} / \mathbf{N}), \Omega^{c}\right)>(2 C)^{-1}} R_{2}(\mathbf{n})\right) .
\end{aligned}
$$

Let $\kappa>0$ be small enough. Suppose that, for some $\mathbf{y} \in f^{\prime}(\Omega)$, we have $d(\mathbf{y}, L) \leqslant \kappa$. Then we may write $\mathbf{y}=\mathbf{b}+\mathbf{h}$, with $\mathbf{b} \in L$ and $\|\mathbf{h}\| \leqslant \kappa$, and thus $d(\omega(\mathbf{y}), K) \leqslant$ $\|\omega(\mathbf{b}+\mathbf{h})-\omega(\mathbf{b})\|$. By the inverse function theorem, we know that $\omega^{\prime}(\mathbf{y})=$ $\left(H_{f}(\omega(\mathbf{y}))\right)^{-1}$ and thus we have $\partial_{j} \omega(\mathbf{y}) \ll \delta^{-1}$ and this implies that $d(\omega(\mathbf{y}), K) \ll$ $\kappa \delta^{-1}$. Finally, we have proved that there exists a constant $C_{p}>0$, which depends only on $C$ and $p$ such that

$$
\begin{aligned}
\mathbf{y} \in f^{\prime}(\Omega) \text { and } d(\mathbf{y}, L) \leqslant \delta / C_{p} & \Longrightarrow d(\omega(\mathbf{y}), K) \leqslant(3 C)^{-1} \\
& \Longrightarrow d\left(\omega(\mathbf{y}), \Omega^{c}\right)>(6 C)^{-1} .
\end{aligned}
$$

Conversely, if $\mathbf{n} / \mathbf{N} \in f^{\prime}(\Omega)$ and if $d\left(\omega(\mathbf{n} / \mathbf{N}), \Omega^{c}\right) \leqslant(2 C)^{-1}$, then $d(\mathbf{n} / \mathbf{N}, L)$ $\gg \delta$ and thus $R_{1}(\mathbf{n}) \ll\left(T \delta^{2}\right)^{-(k-1)}$. In the error term of (5.17), those $\mathbf{n}$ contribute

$$
\sum_{0<d\left(\omega(\mathbf{n} / \mathbf{N}), \Omega^{c}\right) \leqslant(2 C)^{-1}} R_{1}(\mathbf{n}) \ll\left(\prod_{i=1}^{p}\left(1+N_{i}\right)\right)\left(T \delta^{2}\right)^{-(k-1)} .
$$

Now, we give a bound of the contribution of those $\mathbf{n}$ such that $d\left(\omega(\mathbf{n} / \mathbf{N}), \Omega^{c}\right)=0$, that is for $\mathbf{n} / \mathbf{N} \notin f^{\prime}(\Omega)$. For any fixed $\gamma>0$, we have

$$
\sum_{\gamma<d(\mathbf{n} / \mathbf{N}, L) \leqslant 2 \gamma} R_{1}(\mathbf{n}) \ll(1+\gamma)^{p}\left(\prod_{i=1}^{p}\left(1+N_{i}\right)\right)\left((T \gamma)^{-(k-1)}+\left(T \gamma^{2}\right)^{-(k-1)}\right) \text {. }
$$

We take successively the values

$$
\gamma=2^{l} \delta / C_{p}, \quad \text { for } l=0,1,2, \ldots
$$

and we sum the corresponding terms (5.19). Combined with (5.18), this yields

$$
\sum_{d\left(\omega(\mathbf{n} / \mathbf{N}), \Omega^{c}\right) \leqslant(2 C)^{-1}} R_{1}(\mathbf{n}) \ll\left(\prod_{i=1}^{p}\left(1+N_{i}\right)\right)\left(T \delta^{2}\right)^{-(k-1)} .
$$

To complete the bound of the error term in (5.17), we have the obvious

$$
\sum_{d\left(\omega(\mathbf{n} / \mathbf{N}), \Omega^{c}\right)>(2 C)^{-1}} R_{2}(\mathbf{n}) \ll R_{0}\left(\delta^{-2} T^{-(p+1) / 2}+\left(\delta^{6} T\right)^{-(k-1)}\right) .
$$

We insert (5.20) and (5.21) in (5.17) and we obtain (5.11). The proof is complete. 


\subsection{The case with boundary: preliminaries}

We are going to give further hypotheses concerning the compact $D$. Let

$$
\widetilde{D}=\prod_{i=1}^{p}\left[a_{i}, b_{i}\right], \quad \text { with } \frac{1}{C} \leqslant a_{i}<b_{i} \leqslant C \text { for } i=1, \ldots, p,
$$

and we suppose that

$$
K \subset \prod_{i=1}^{p}\left[a_{i}-\frac{1}{3 C}, b_{i}+\frac{1}{3 C}\right] \subset \Omega \subset\left[\frac{1}{3 C}, 2 C\right]^{p} .
$$

Now, we write systematically, for $\mathbf{x} \in \mathbb{R}^{p}$

$$
\mathbf{x}^{\prime}=\left(x_{1}, \ldots, x_{p-1}\right) \quad \text { and } \quad \mathbf{x}=\left(\mathbf{x}^{\prime}, x_{p}\right) .
$$

The more interesting case is when $D$ has the shape :

$$
\left\{\begin{array}{l}
D=\left\{\left(\mathbf{x}^{\prime}, x_{p}\right) \mid \mathbf{x}^{\prime} \in D^{\prime} \text { and } a_{p} \leqslant x_{p} \leqslant \varphi\left(\mathbf{x}^{\prime}\right)\right\} \\
\text { with } \varphi: \Omega^{\prime} \rightarrow\left[a_{p}, b_{p}\right], \text { a regular function. }
\end{array}\right.
$$

Here, we have set

$$
\left\{\begin{array}{l}
D^{\prime}=\prod_{i=1}^{p-1}\left[a_{i}, b_{i}\right] \subset \mathbb{R}^{p-1}, \\
\Omega^{\prime}=\left\{\mathbf{x}^{\prime} \in \mathbb{R}^{p-1} \mid\left(\mathbf{x}^{\prime}, x_{p}\right) \in \Omega \text { for at least one } x_{p} \in \mathbb{R}\right\} .
\end{array}\right.
$$

Taking a finite union of such domains, and reversing the coordinates for some of them, we cover all known applications.

Our study of the $B$-transformation with boundary is as follows. Firstly, we treat the case where $D$ is an hyper-rectangle, for which our method is quite relevant. For this, we boil down to the case without boundary by Lemma 5.2.3. of [4], which involves the orthogonality of complex exponential functions. Secondly, we boil down the general case (5.24) to the previous one by means of lemma 5.2.4. of [4], which involves Mellin's transformation (or Perron's formula). Our result is suddenly less efficient: our error term is considerably larger and the main term is much more complicated. This is however sufficient to improve on Krätzel's theorems, both on the error term and on the simplification of the hypotheses.

We recall them briefly.

Krätzel's theorems: In his systematic study of the multidimensional $B$ process in [5], Krätzel treats directly the important case where $\operatorname{det} H_{f}(\mathbf{x})$ may be abnormally small, in Theorem 2.24 of [5], for $p=2$, and Theorem 2.28 of [5] for $p \geqslant 3$. In both theorems, the more difficult problem of exponential sums with boundary is considered, the compact domain $D$ satisfying (5.24). As for the hypotheses, only partial derivatives up to the third order are involved, but, for $p=2$ on the one hand, and especially for $p \geqslant 3$ on the other hand, a lot 
of complicated conditions are needed for technical reasons, among which is the injectivity of $f^{\prime}$. Furthermore, the partial determinants

$$
\Delta_{j}(\mathbf{x})=\left|\begin{array}{ccc}
\partial_{1}^{2} f(\mathbf{x}) & \ldots & \partial_{1} \partial_{j} f(\mathbf{x}) \\
\vdots & \ddots & \vdots \\
\partial_{j} \partial_{1} f(\mathbf{x}) & \cdots & \partial_{j}^{2} f(\mathbf{x})
\end{array}\right|, \quad \text { with } j=1,2, \ldots, p
$$

which are related to the proof that iterates the one-dimensional case, require a lower bound, say

$$
\left|\Delta_{j}(\mathbf{x})\right| \geqslant \delta_{j}>0, \quad \text { for } j=1,2, \ldots, p \text { and } \mathbf{x} \in \Omega .
$$

Here, $\delta_{p}$ corresponds to our $\delta$.

These results may be stated as

$$
S_{D}=\text { main term }+E \text {. }
$$

In the standard situation, and with our notations, theorems 2.24 and 2.28 of [5] cannot do better than

$$
E \ll M_{1} \cdots M_{p-1} \mathcal{L}
$$

where we have set

$$
\mathcal{L}=\log \left(M_{1}+\ldots+M_{p}+T\right)
$$

and, under some natural conditions, this bound is reached in formula (2.79) of [5], in the case $p=2$, and in formula $(2.88)$ of $[\mathrm{K}]$ in the case $p \geqslant 3$.

Note that in the applications, this error term is widely sharp enough for $p=2$. But when $p$ increases, it becomes more and more awkward.

For comparison, our Theorem 5.3 gives

$$
E \ll M_{1} \cdots M_{p-1} \frac{M_{p}^{1 / 2}}{T^{1 / 2}} \mathcal{L}+\text { secondary error terms },
$$

and this is better, for, in the applications, $T$ is greater than $M_{p}$ (but smaller than $\left.M_{p}^{2}\right)$.

\subsection{The $B$-process with boundary: the case of an hyper-rectangle}

Proposition 5.2. ${ }^{4}$ Let the hypotheses and notations of section 5.1 hold. For $\vartheta \in[0,1]^{p}$, we set

$$
Z_{D}(\vartheta)=\sum_{\substack{\mathbf{m} / \mathbf{M} \in D \\ \mathbf{m} \in \mathbb{Z}^{p}}} e(\langle\vartheta, \mathbf{m}\rangle)
$$

and let

$$
\mu(D)=\int_{[0,1]^{p}}\left|Z_{D}(\vartheta)\right| d \vartheta .
$$

\footnotetext{
${ }^{4}$ Here again we increase the counter to fit with Sargos' numbering.
} 
We then have

$$
\begin{aligned}
S_{D}= & \frac{M_{1} \cdots M_{p}}{T^{p / 2}} e^{i \pi \sigma / 4} \\
& \times \int_{[0,1]^{p}}\left(\sum_{\mathbf{n} \in \mathbb{Z}^{p}} \frac{\chi(\omega((\mathbf{n}+\vartheta) / \mathbf{N}))}{\left|\operatorname{det} H_{f}\left(\omega\left(\frac{\mathbf{n}+\vartheta}{\mathbf{N}}\right)\right)\right|^{1 / 2}} e\left(T f^{*}\left(\frac{\mathbf{n}+\vartheta}{\mathbf{N}}\right)\right)\right) Z_{D}(\vartheta) d \vartheta \\
& +O\left(\left(\prod_{i=1}^{p}\left(T+M_{i}\right)\right) \mu(D) T^{-(p+1) / 2} \delta^{-2}\right) \\
& +O\left(\left(\prod_{i=1}^{p}\left(T+M_{i}\right)\right) \mu(D)\left(T \delta^{6}\right)^{-(k-1)}\right)
\end{aligned}
$$

the implied constants depending at most on $k$ and $C$.

In particular, if $D$ is an hyper-rectangle, say $D=\prod_{i=1}^{p}\left[a_{i}, b_{i}\right]$ with $\max _{i} \mid b_{i}-$ $a_{i} \mid \ll 1$, then we have

$$
\mu(D) \ll \mathcal{L}^{p}
$$

\section{Remarks.}

3. The final goal of the study of the exponential sum $S_{D}$ is always to give a bound, the $B$-process being an intermediary step. So, Proposition 5.2 will be used mainly in the form of the following bound:

$$
\begin{aligned}
S_{D} \ll & \frac{M_{1} \cdots M_{p}}{T^{p / 2}} \mu(D) \\
& \times \max _{\vartheta \in[0,1]^{p}}\left|\sum_{\mathbf{n} \in \mathbb{Z}^{p}} \frac{\chi(\omega((\mathbf{n}+\vartheta) / \mathbf{N}))}{\left|\operatorname{det} H_{f}(\omega((\mathbf{n}+\vartheta) / \mathbf{N}))\right|^{1 / 2}} e\left(T f^{*}\left(\frac{\mathbf{n}+\vartheta}{\mathbf{N}}\right)\right)\right| \\
& +\left(\prod_{i=1}^{p}\left(T+M_{i}\right)\right) \mu(D) T^{-(p+1) / 2} \delta^{-2} \\
& +\left(\prod_{i=1}^{p}\left(T+M_{i}\right)\right) \mu(D)\left(T \delta^{6}\right)^{-(k-1)}
\end{aligned}
$$

If we have to bound a sum of exponential sums that depend on a parameter $h$

$$
\sum_{h=1}^{H} S_{D}(h)=\sum_{h=1}^{H} \sum_{\mathbf{m} \in D \cap \mathbb{Z}^{p}} \chi_{h}\left(\frac{m_{1}}{M_{1}}, \ldots, \frac{m_{p}}{M_{p}}\right) e\left(T f_{h}\left(\frac{m_{1}}{M_{1}}, \ldots, \frac{m_{p}}{M_{p}}\right)\right)
$$

the compact $D$ and the open $\Omega$ being independent of $h$, all the hypotheses 
being uniform in $h$, then we have

$$
\begin{aligned}
& \sum_{h=1}^{H} S_{D}(h) \ll \frac{M_{1} \cdots M_{p}}{T^{p / 2}} \mu(D) \\
& \quad \times \max _{\vartheta \in[0,1]^{p}}\left|\sum_{h=1}^{H} \sum_{\mathbf{n} \in \mathbb{Z}^{p}} \frac{\chi_{h}\left(\omega_{h}((\mathbf{n}+\vartheta) / \mathbf{N})\right)}{\left|\operatorname{det} H_{f_{h}}\left(\omega_{h}((\mathbf{n}+\vartheta) / \mathbf{N})\right)\right|^{1 / 2}} e\left(T f_{h}^{*}\left(\frac{\mathbf{n}+\vartheta}{\mathbf{N}}\right)\right)\right| \\
& \quad+H\left(\prod_{i=1}^{p}\left(T+M_{i}\right)\right) \mu(D) T^{-(p+1) / 2} \delta^{-2} \\
& \quad+H\left(\prod_{i=1}^{p}\left(T+M_{i}\right)\right) \mu(D)\left(T \delta^{6}\right)^{-(k-1)} .
\end{aligned}
$$

Such a situation occurs in lattice point problems (cf. [5] or [4]), where the summation over $h$ is split in intervals of the form $H+1, \ldots, 2 H$, while the phase function is now $f_{h}(\mathbf{x})=\frac{h}{H} f(\mathbf{x})$. But in practice, it is difficult to use the parameter $h$ as a true variable.

4. The formulas (5.27) and (5.28) show that Proposition 5.2 is indissociable of a bound for $\mu(D)$. In the case where $D=\prod_{i=1}^{p}\left[a_{i}, b_{i}\right]$ with $b_{i}-a_{i} \ll 1$ (as $\Omega$ is bounded, this condition is not restrictive), we have the obvious bound

$$
\mu(D) \ll \mathcal{L}^{p},
$$

where $\mathcal{L}$ is defined in (5.25). Unfortunately, when $D$ is defined as in (5.24), we did not succeed in finding a satisfactory bound for $\mu(D)$.

Proof of Proposition 5.2. First of all, we state an obvious lemma which is an immediate consequence of the orthogonality property of the complex exponential functions.

Lemma 5.1. Let $a$ and $b: \mathbb{Z}^{p} \longrightarrow \mathbb{C}$ be two mappings, a having a bounded support. For any compact set $D$ of $\mathbb{R}^{p}$, we have

$$
\sum_{\substack{\mathbf{m} / \mathbf{M} \in D \\ \mathbf{m} \in \mathbb{Z}^{p}}} a(\mathbf{m}) b(\mathbf{m})=\int_{[0,1]^{p}}\left(\sum_{\mathbf{m} \in \mathbb{Z}^{p}} a(\mathbf{m}) e(-\langle\vartheta, \mathbf{m}\rangle)\right)\left(\sum_{\mathbf{n} \in D \cap \mathbb{Z}^{p}} b(\mathbf{n}) e(\langle\vartheta, \mathbf{n}\rangle)\right) d \vartheta .
$$

Now, we take

$$
a(\mathbf{m})=\chi\left(\frac{\mathbf{m}}{\mathbf{M}}\right) e\left(T f\left(\frac{\mathbf{m}}{\mathbf{M}}\right)\right) \quad \text { and } \quad b(\mathbf{n})=1,
$$

so that (5.30) becomes

$$
S_{D}=\int_{[0,1]^{p}} S(\vartheta) Z_{D}(\vartheta) d \vartheta
$$


with

$$
S(\vartheta)=\sum_{\mathbf{m} \in \mathbb{Z}^{p}} \chi\left(\frac{\mathbf{m}}{\mathbf{M}}\right) e\left(T f_{\vartheta}\left(\frac{\mathbf{m}}{\mathbf{M}}\right)\right)
$$

and

$$
f_{\vartheta}(\mathbf{x})=f(\mathbf{x})-\left\langle\frac{\vartheta}{\mathbf{N}}, \mathbf{x}\right\rangle .
$$

We apply Theorem 5.2 to the sum without boundary $S(\vartheta)$. For this, we have to use (5.10) to give a bound uniform in $\vartheta$ for $R$. Furthermore, we note that

$$
f_{\vartheta}^{*}\left(\frac{\mathbf{n}}{\mathbf{N}}\right)=f^{*}\left(\frac{\mathbf{n}+\vartheta}{\mathbf{N}}\right)
$$

Finally, we have obtained exactly (5.26).

\subsection{The $B$-process with a regular boundary}

All the hypotheses are those of section 5.1. Moreover, we assume that the compact $D$ satisfies the regularity condition (5.24). We introduce the notation, for any $\varepsilon>0$

$$
\begin{aligned}
\Re(\varphi, \varepsilon) & =\#\left\{\mathbf{m}^{\prime} \in D_{0}^{\prime} \cap \mathbb{Z}^{p} \mid d\left(M_{p} \varphi\left(\frac{m_{1}}{M_{1}}, \ldots, \frac{m_{p-1}}{M_{p-1}}\right), m_{p}\right) \leqslant \varepsilon\right\}, \\
D_{0}^{\prime} & =\prod_{i=1}^{p-1}\left[M_{i} a_{i}, M_{i} b_{i}\right],
\end{aligned}
$$

which denotes the number of lattice points within a distance $\varepsilon$ to the hypersurface

$$
\left\{\mathbf{x} \in \mathbb{R}^{p} \mid \mathbf{x}^{\prime} \in D_{0}^{\prime} \text { and } x_{p}=M_{p} \varphi\left(\frac{x_{1}}{M_{1}}, \ldots, \frac{x_{p-1}}{M_{p-1}}\right)\right\} .
$$

The problem of estimating or of giving upper bounds for $\Re(\varphi, \varepsilon)$ is well known and is the aim of Kratzel's book [5] (see also [4] for the best known results in the case $p=2)$. The expected bound, at least if $\varepsilon$ is not too small, and if $\operatorname{det} H_{\varphi}\left(\mathbf{x}^{\prime}\right)$ does not vanish, is

$$
\Re(\varphi, \varepsilon) \ll \varepsilon M_{1} \cdots M_{p-1},
$$

Now we introduce the new phase function. For any real $t$, we set

$$
u(\mathbf{x})=\frac{\varphi\left(\mathbf{x}^{\prime}\right)}{x_{p}}, \quad f_{t}(\mathbf{x})=f(\mathbf{x})+\frac{t}{2 \pi T} \log u(\mathbf{x}), \quad \text { and } \quad \chi_{1}(\mathbf{x})=\chi(\mathbf{x}) u(\mathbf{x}) .^{5}
$$

This defines a function $f_{t}: \Omega \rightarrow \mathbb{R}$. If $t$ is not too large, we are in the situation of section 3.2. In particular, we have the following property.

\footnotetext{
${ }^{5}$ It seems necessary to assume henceforth the harmless condition that $\varphi$ is $C^{k}$ on $\overline{\Omega^{\prime}}$.
} 
Lemma 5.2. There exists a real number $\eta_{0}>0$ that depends only on $k$ and $C$, such that, for

$$
|t| \leqslant X_{0}, \quad \text { with } X_{0}=\eta_{0} \delta T,
$$

the function $f_{t}$ is in $C^{k}(\Omega, \mathbb{R})$ and satisfies

$$
\left|\operatorname{det} H_{f_{t}}(\mathbf{x})\right| \geqslant \delta / 2 \quad \text { and } \quad\left|\partial^{\alpha} f_{t}(\mathbf{x})\right| \leqslant 2 C, \quad \text { for } \quad|\alpha| \leqslant k \quad \text { and } \mathbf{x} \in \Omega \text {, }
$$

and such that $f_{t}^{\prime}$ is injective in an open connected set $\Omega_{0}$ containing $K+B(\mathbf{0}, 1 / 6 C)$.

Proof. Everything in this lemma is obvious, except the injectivity of $f_{t}^{\prime}$ that we are going to prove. In order to simplify, we set $\eta=t /(2 \pi T)$, so that $f_{t}(\mathbf{x})=$ $f(\mathbf{x})+\eta u(\mathbf{x})$. We have to show that $f_{t}^{\prime}$ is injective in $\Omega_{0}$, provided that $\eta \delta^{-1}$ is small enough. Suppose that we have

$$
f_{t}^{\prime}(\mathbf{x}+\mathbf{h})=f_{t}^{\prime}(\mathbf{x}), \quad \text { for some } \mathbf{x} \text { and } \mathbf{x}+\mathbf{h} \in \Omega_{0},
$$

that is

$$
f^{\prime}(\mathbf{x}+\mathbf{h})-f^{\prime}(\mathbf{x})=\mathbf{O}(\eta\|\mathbf{h}\|),
$$

where we have written $\mathbf{O}$ instead of $O$ because the equality holds in $\mathbb{R}^{p}$. But, for $\eta \delta^{-1}$ small enough, Lemma 4.3 shows that this is not possible for $\mathbf{h} \neq \mathbf{0}$, and the lemma is proved.

At last, we define

$$
\omega_{t}: f_{t}^{\prime}\left(\Omega_{0}\right) \rightarrow \Omega_{0}
$$

as being the inverse map of the restriction of $f_{t}^{\prime}$ to $\Omega_{0}$.

We are now ready to state the main result of this paper.

Theorem 5.3. We assume that the above notations and hypotheses hold. Then, for any positive real numbers $\varepsilon$ and $X \leqslant X_{0}$, we have

$$
\begin{aligned}
S_{D}= & \frac{M_{1} \cdots M_{p} e^{i \pi \sigma / 4}}{2 \pi T^{p / 2}} \int_{-X}^{X} \int_{[0,1]^{p}} \widetilde{S}(t, \vartheta) Z_{\widetilde{D}}(\vartheta) d \vartheta \frac{d t}{1+i t} \\
& +O\left(\left(\prod_{i=1}^{p}\left(T+M_{i}\right)\right)\left(T^{-(p+1) / 2} \delta^{-2}+\left(T \delta^{6}\right)^{-(k-1)}\right) \mathcal{L}^{p+1}\right) \\
& +O(\Re(\varphi, \varepsilon) \mathcal{L})+O\left(\frac{M_{1} \cdots M_{p}}{\varepsilon X}\right)+O\left(\frac{M_{1} \cdots M_{p}}{X} \mathcal{L}\right),
\end{aligned}
$$

where we have set

$$
\widetilde{S}(t, \vartheta)=\sum_{\mathbf{n} \in \mathbb{Z}^{p}} \frac{\chi_{1}\left(\omega_{t}((\mathbf{n}+\vartheta) / \mathbf{N})\right)}{\left|\operatorname{det} H_{f_{t}}\left(\omega_{t}((\mathbf{n}+\vartheta) / \mathbf{N})\right)\right|^{1 / 2}} e\left(T f_{t}^{*}\left(\frac{n+\vartheta}{\mathbf{N}}\right)\right) .
$$




\section{Remarks.}

5. For the convenience of the reader, and for a quick reading of this result, we recall some facts concerning the notation. First, $S_{D}$ is the exponential sum defined in (5.2), while $D$ is the compact whose regular boundary satisfies (5.24), as in Krätzel's theorems. As for $Z_{\widetilde{D}}(\vartheta)$, we have defined $Z_{D}(\vartheta)$ in the course of Proposition 5.2 and $\widetilde{D}$ is defined in (5.22); but the only fact to keep in mind is that we have the bound

$$
\int_{[0,1]^{p}}\left|Z_{\widetilde{D}}(\vartheta)\right| d \vartheta \ll \mathcal{L}^{p}
$$

In practice, this amounts to increase the main term by a factor $\mathcal{L}^{p}$. A trivial bound of the multiple exponential sum $\widetilde{S}(t, \vartheta)$ yields

$$
\text { main term } \ll M_{1} \cdots M_{p}\left(\prod_{i=1}^{p}\left(\frac{T}{M_{i}^{2}}\right)^{1 / 2} \mathcal{L}^{p+1}\right)
$$

which is, up to the factor $\mathcal{L}^{p+1}$, the bound expected for the van der Corput's inequality.

The first error term is the same as above, but divided by $T^{1 / 2}$ and poses no problem. The following error term, involving $\left(T \delta^{6}\right)^{-(k-1)}$, is negligible in practice, because $k$ may be taken as large as required, while $\delta$ will never be too small.

At last, the error terms $O(\Re(\varphi, \varepsilon) \mathcal{L})+O\left(M_{1} \cdots M_{p} / \varepsilon X\right)$ are the main ones. An optimization on $\varepsilon$ has to be done. If we have the expected bound (5.33), then we may take $X \sim \delta T$ and $\varepsilon=\left(M_{p} / \delta T \mathcal{L}\right)^{1 / 2}$ and these error terms become

$$
O\left(\frac{M_{1} \cdots M_{p}}{\left(\delta T M_{p}\right)^{1 / 2}} \mathcal{L}^{1 / 2}\right)
$$

so that the best possible saving is a factor $\mathcal{L} /\left(\delta T M_{p}\right)^{1 / 2}$.

6. The natural multidimensional van der Corput's transformation should be written as

$$
S_{D}=M_{1} \cdots M_{p} e^{i \pi \sigma / 4} \sum_{\mathbf{n} / \mathbf{N} \in f^{\prime}(D)} \frac{\chi(\omega(\mathbf{n} / \mathbf{N})) e\left(T f^{*}(\mathbf{n} / \mathbf{N})\right)}{\left|\operatorname{det} H_{f}(\omega(\mathbf{n} / \mathbf{N}))\right|^{1 / 2} T^{p / 2}}+E .
$$

So are Krätzel's results. Our main term is, of course, more intricate. But our new exponential sum $\widetilde{S}(t, \vartheta)$ is without boundary, and the new phase function $f_{t}^{*}$ is similar to $f^{*}$, according to proposition 3.2 . So the complexity of our main term has some compensations, not only in the error term.

Proof of Theorem 5.3. We begin with a classical lemma, namely the truncated Perron's formula. 
Lemma 5.3. Let $a, c$ and $X$ be three positive numbers, $a \neq 1$. We have

$$
\frac{1}{2 i \pi} \int_{c-i X}^{c+i X} a^{s} \frac{d s}{s}=\left\{\begin{array}{lll}
0 & \text { if } & a<1 \\
1 & \text { if } & a>1
\end{array}+O\left(\frac{a^{c}}{X|\log a|}\right)+O\left(\frac{a^{c}}{X}\right)\right.
$$

the implied constants being absolute.

We come now to the proof of Theorem 5.3. We set

$$
\varrho(\mathbf{m})=\varrho\left(\mathbf{m}^{\prime}, m_{p}\right)=\chi\left(\frac{\mathbf{m}}{\mathbf{M}}\right) e\left(T f\left(\frac{\mathbf{m}}{\mathbf{M}}\right)\right)
$$

and

$$
\frac{\mathbf{m}^{\prime}}{\mathbf{M}^{\prime}}=\left(\frac{m_{1}}{M_{1}}, \ldots, \frac{m_{p-1}}{M_{p-1}}\right)
$$

for $\mathbf{m} \in \mathbb{Z}^{p}$.

For any positive real numbers $\varepsilon$ and $X \leqslant X_{0}$, recalling that $\widetilde{D}$ is defined in (5.22), we have

$$
\begin{aligned}
& \frac{1}{2 i \pi} \int_{1-i X}^{1+i X}\left(\sum_{\mathbf{m} / \mathbf{M} \in \widetilde{D}} \varrho\left(\mathbf{m}^{\prime}, m_{p}\right)\left(\frac{M_{p} \varphi\left(\mathbf{m}^{\prime} / \mathbf{M}^{\prime}\right)}{m_{p}}\right)^{s}\right) \frac{d s}{s} \\
& =\sum_{\substack{\mathbf{m} / \mathbf{M} \in \widetilde{D} \\
\left|m_{p}-M_{p} \varphi\left(\mathbf{m}^{\prime} / \mathbf{M}^{\prime}\right)\right| \leqslant \varepsilon}} \varrho\left(\mathbf{m}^{\prime}, m_{p}\right) \frac{1}{2 i \pi} \int_{1-i X}^{1+i X}\left(\frac{M_{p} \varphi\left(\mathbf{m}^{\prime} / \mathbf{M}^{\prime}\right)}{m_{p}}\right)^{s} \frac{d s}{s} \\
& \quad+\sum_{\substack{\mathbf{m} / \mathbf{M} \in \widetilde{D} \\
\left|m_{p}-M_{p} \varphi\left(\mathbf{m}^{\prime} / \mathbf{M}^{\prime}\right)\right|>\varepsilon}} \varrho\left(\mathbf{m}^{\prime}, m_{p}\right) \frac{1}{2 i \pi} \int_{1-i X}^{1+i X}\left(\frac{M_{p} \varphi\left(\mathbf{m}^{\prime} / \mathbf{M}^{\prime}\right)}{m_{p}}\right)^{s} \frac{d s}{s}
\end{aligned}
$$

As the bound $\varrho(\mathbf{m}) \ll 1$ holds for each $\mathbf{m} \in \mathbb{Z}^{p}$, and as we have

$$
\frac{1}{2 i \pi} \int_{1-i X}^{1+i X}\left(\frac{M_{p} \varphi\left(\frac{\mathbf{m}^{\prime}}{\mathbf{M}^{\prime}}\right)}{m_{p}}\right) \frac{d s}{s} \ll \mathcal{L}
$$

the first term in the right member above is

$$
\ll \Re(\varphi, \varepsilon) \mathcal{L} .
$$

For the last term in (5.40), we apply the truncated Perron's formula, and we 
get

$$
\begin{aligned}
\sum_{\substack{\mathbf{m} / \mathbf{M} \in \widetilde{D} \\
\left|m_{p}-M_{p} \varphi\left(\mathbf{m}^{\prime} / \mathbf{M}^{\prime}\right)\right|>\varepsilon}} \varrho\left(\mathbf{m}^{\prime}, m_{p}\right) \frac{1}{2 i \pi} \int_{1-i X}^{1+i X}\left(\frac{M_{p} \varphi\left(\mathbf{m}^{\prime} / \mathbf{M}^{\prime}\right)}{m_{p}}\right)^{s} \frac{d s}{s} \\
=\sum_{\substack{\mathbf{m} / \mathbf{M} \in \widetilde{D} \\
m_{p}<M_{p} \varphi\left(\frac{\mathbf{m}^{\prime}}{\mathbf{M}^{\prime}}\right)-\varepsilon}} \varrho\left(\mathbf{m}^{\prime}, m_{p}\right) \\
+\sum_{\substack{\mathbf{m} / \mathbf{M} \in \widetilde{D} \\
\left|m_{p}-M_{p} \varphi\left(\frac{\mathbf{m}^{\prime}}{\mathbf{M}^{\prime}}\right)\right|>\varepsilon}} O\left(\left(X \log \left(\frac{M_{p} \varphi\left(\mathbf{m}^{\prime} / \mathbf{M}^{\prime}\right)}{m_{p}}\right)\right)^{-1}\right) .
\end{aligned}
$$

The first term in the right hand side of (5.41) is

$$
\begin{aligned}
& \sum_{\substack{\mathbf{m} / \mathbf{M} \in \widetilde{D} \\
m_{p}<M_{p} \varphi\left(\mathbf{m}^{\prime} / \mathbf{M}^{\prime}\right)-\varepsilon}} \varrho(\mathbf{m}) \\
& =\sum_{\substack{\mathbf{m} / \mathbf{M} \in \widetilde{D} \\
m_{p} \leqslant M_{p} \varphi\left(\mathbf{m}^{\prime} / \mathbf{M}^{\prime}\right) \\
=}} \varrho(\mathbf{m})-\sum_{S_{D}+O(\Re(\varphi, \varepsilon)) .} \varrho(\mathbf{m}) \\
& M_{p} \varphi\left(\mathbf{m}^{\prime} / \mathbf{M}^{\prime}\right)-\varepsilon \leqslant m_{p} \leqslant M_{p} \varphi\left(\mathbf{m}^{\prime} / \mathbf{M}^{\prime}\right)
\end{aligned}
$$

We come back to the left hand side of (5.40) in which we take $s=1+i t$. We have

$$
\begin{aligned}
\sum_{\mathbf{m} / \mathbf{M} \in \widetilde{D}} \varrho\left(\mathbf{m}^{\prime}, m_{p}\right)\left(\frac{M_{p} \varphi\left(\mathbf{m}^{\prime} / \mathbf{M}^{\prime}\right)}{m_{p}}\right)^{s} & =\sum_{\mathbf{m} / \mathbf{M} \in \widetilde{D}} \varrho(\mathbf{m}) u\left(\frac{\mathbf{m}}{\mathbf{M}}\right) e\left(\frac{t}{2 \pi} \log u\left(\frac{\mathbf{m}}{\mathbf{M}}\right)\right) \\
& =\sum_{\mathbf{m} / \mathbf{M} \in \widetilde{D}} \chi_{1}\left(\frac{\mathbf{m}}{\mathbf{M}}\right) e\left(T f_{t}\left(\frac{\mathbf{m}}{\mathbf{M}}\right)\right) .
\end{aligned}
$$

Now, we combine all these formulas to get

$$
\begin{aligned}
S_{D}= & \frac{1}{2 i \pi} \int_{1-i X}^{1+i X}\left(\sum_{\mathbf{m} / \mathbf{M} \in \widetilde{D}} \chi_{1}\left(\frac{\mathbf{m}}{\mathbf{M}}\right) e\left(T f_{t}\left(\frac{\mathbf{m}}{\mathbf{M}}\right)\right)\right) \frac{d s}{s}+O(\Re(\varphi, \varepsilon)) \\
& +\sum_{\substack{\mathbf{m} / \mathbf{M} \in \widetilde{D} \\
\left|m_{p}-M_{p} \varphi\left(\mathbf{m}^{\prime} / \mathbf{M}^{\prime}\right)\right|>\varepsilon}} O\left(\left(X \log \left(\frac{M_{p} \varphi\left(\mathbf{m}^{\prime} / \mathbf{M}^{\prime}\right)}{m_{p}}\right)\right)^{-1}\right) .
\end{aligned}
$$

The main term is the first one in the right hand side of (5.44). The inner sum 
may be transformed by Proposition 5.2, and this gives

$$
\begin{aligned}
\frac{1}{2 i \pi} \int_{1-i X}^{1+i X}\left(\sum_{\mathbf{m} / \mathbf{M} \in \widetilde{D}} \chi_{1}\left(\frac{\mathbf{m}}{\mathbf{M}}\right) e\left(T f_{t}\left(\frac{\mathbf{m}}{\mathbf{M}}\right)\right)\right) \frac{d s}{s} \\
=\frac{M_{1} \cdots M_{p} e^{i \pi \sigma / 4}}{2 \pi T^{p / 2}} \int_{-X}^{X} \int_{[0,1]^{p}} \widetilde{S}(t, \vartheta) Z_{\widetilde{D}}(\vartheta) d \vartheta \frac{d t}{1+i t} \\
\quad+O\left(\left(\prod_{i=1}^{p}\left(T+M_{i}\right)\right)\left(T^{-(p+1) / 2} \delta^{-2}+\left(T \delta^{6}\right)^{-(k-1)}\right) \mathcal{L}^{p+1}\right) .
\end{aligned}
$$

Now, it remains only to bound the error term

$$
R=\sum_{\substack{\mathbf{m} / \mathbf{M} \in \widetilde{D} \\\left|m_{p}-M_{p} \varphi\left(\mathbf{m}^{\prime} / \mathbf{M}^{\prime}\right)\right|>\varepsilon}} O\left(\left(X \log \left(\frac{M_{p} \varphi\left(\mathbf{m}^{\prime} / \mathbf{M}^{\prime}\right)}{m_{p}}\right)\right)^{-1}\right) .
$$

For this, we first note that elementary calculation yields the inequality

$$
\left(\log \left(\frac{M_{p} \varphi\left(\mathbf{m}^{\prime} / \mathbf{M}^{\prime}\right)}{m_{p}}\right)\right)^{-1} \ll \frac{M_{p}}{\left|m_{p}-M_{p} \varphi\left(\mathbf{m}^{\prime} / \mathbf{M}^{\prime}\right)\right|} .
$$

Moreover, the term $R$ is obtained on summing both over $\mathbf{m}^{\prime}$ and $m_{p}$. For each $\mathbf{m}^{\prime}$, we define

$$
R\left(\mathbf{m}^{\prime}\right)=X^{-1} \sum_{\substack{M_{p} a_{p} \leqslant m_{p} \leqslant M_{p} b_{p} \\\left|m_{p}-M_{p} \varphi\left(\mathbf{m}^{\prime} / \mathbf{M}^{\prime}\right)\right|>\varepsilon}} \frac{M_{p}}{\left|m_{p}-M_{p} \varphi\left(\mathbf{m}^{\prime} / \mathbf{M}^{\prime}\right)\right|},
$$

so that we have

$$
R \ll \sum_{\mathbf{m}^{\prime} \in D_{0}^{\prime}} R\left(\mathbf{m}^{\prime}\right)
$$

Define $\nu\left(\mathbf{m}^{\prime}\right)$ as being the integer $m_{p}$ which minimizes $\left|m_{p}-M_{p} \varphi\left(\mathbf{m}^{\prime} / \mathbf{M}^{\prime}\right)\right|$. With this notation, we have

$$
R\left(\mathbf{m}^{\prime}\right) \ll X^{-1} \frac{M_{p}}{\varepsilon+\left|\nu\left(\mathbf{m}^{\prime}\right)-M_{p} \varphi\left(\mathbf{m}^{\prime} / \mathbf{M}^{\prime}\right)\right|}+X^{-1} M_{p} \sum_{1 \leqslant h \ll M_{p}} \frac{1}{h} .
$$

Summing over $\mathbf{m}^{\prime}$ yields

$$
R \ll \sum_{\mathbf{m}^{\prime} \in D_{0}^{\prime}} R\left(\mathbf{m}^{\prime}\right) \ll \frac{M_{1} \cdots M_{p-1} M_{p}}{X \varepsilon}+\frac{M_{1} \cdots M_{p}}{X} \mathcal{L} .
$$

We transfer (5.45) and (5.46) in (5.44) and we get exactly (5.35). The theorem is proved. 


\subsection{A bound for $\Re(\varphi, \varepsilon)$}

This section is left void.

\section{Appendix}

We reproduce here a short note left by the Author, where he comments on Theorem 4.1 and describes a further development which he unfortunately did not have time to write up in full detail.

\section{Une curiosité}

La démonstration du Théorème 1 permet d'obtenir une estimation de l'intégrale oscillante

$$
I_{\gamma}(\tau)=\int_{\gamma} \chi(\mathbf{x}) e(\tau f(\mathbf{x}) d \mathbf{x}
$$

avec les mêmes hypothèses que précédemment, mais lorsque l'intégrale s'effectue sur un cône $\gamma$, ayant son sommet à l'origine, et ayant une propriété de "compatibilité avec $H_{f}(\mathbf{0})$ ", de sorte que $\mathbf{0}$ soit à la fois un point critique non dégénéré de la phase $f$ et un point fortement singulier pour $\gamma$. Le cas où $\gamma$ est un demi-espace a été complètement étudié dans $[\mathrm{H}]$. Pour décrire $\gamma$ ainsi que le résultat obtenu, il faut introduire des notations.

Soit $A$ la matrice symétrique réelle $p \times p$ égale à $H_{f}(\mathbf{0})$. Soit $\left\{\lambda_{1}, \ldots, \lambda_{m}\right.$, $\left.-\mu_{1}, \ldots-\mu_{n}\right\}$ l'ensemble des valeurs propres de $A$, comptées avec leur multiplicité, de sorte que $m+n=p$ (et $\sigma=m-n$, où $\sigma$ est la signature de $A$ ). On suppose que chaque $\lambda_{i}$ et chaque $\mu_{j}$ est positif. Soit $E^{+}$(resp. $E^{-}$) le sous-espace de dimension $m$ (resp. $n$ ) engendré par les vecteurs propres associés aux valeurs propres positives (resp. négatives); soit $S^{+}$(resp. $S^{-}$) la sphère unité de l'espace $E^{+}$(resp. $E^{-}$) pour la norme induite par la norme euclidienne de l'espace ambiant.

Soient enfin $K^{+}$un compact de $S^{+}$, dont l'aire $(m-1)$-dimensionnelle est notée $S_{m-1}\left(K^{+}\right)$, et $K^{-}$un compact de $S^{-}$dont l'aire $(n-1)$-dimensionnelle est notée $S_{n-1}\left(K^{-}\right)$. On définit alors le cône

$$
\gamma=\mathbb{R}_{+} K^{+}+\mathbb{R}_{+} K^{-}=\left\{r \mathbf{u}+s \mathbf{v} \mid r \geqslant 0, s \geqslant 0, \mathbf{u} \in K^{+}, \mathbf{v} \in K^{-}\right\},
$$

ainsi que la constante

$$
G_{\gamma}=\frac{\Gamma(m / 2) \Gamma(n / 2) S_{m-1}\left(K^{+}\right) S_{n-1}\left(K^{-}\right)}{4 \pi^{p / 2}} .
$$

Nous dirons qu'un cône $\gamma$ de la forme (8) est "compatible avec $H_{f}(\mathbf{0})$ ". Nous énonçons notre résultat sans nous soucier d'uniformité.

Théorème 2. Soit $\Omega$ un ouvert de $\mathbb{R}^{p}$ contenant l'origine. Soit $f \in C^{p+5}(\Omega, \mathbb{R})$ une fonction possédant un point critique non dégénéré à l'origine. Soit également un cône $\gamma$ compatible avec $H_{f}(0)$. 
Alors il existe un voisinage $V$ de l'origine suffisamment petit, ne dépendant que de $f$, tel que, pour toute fonction test $\chi \in C_{0}^{p+5}(V, \mathbb{C})$, on ait

$$
I_{\gamma}(\tau)=\frac{G_{\gamma} e^{\sigma i \pi / 4} \chi(\mathbf{0}) e(\tau f(\mathbf{0}))}{\left|\operatorname{det} H_{f}(\mathbf{0})\right|^{1 / 2} \tau^{p / 2}}+O\left(\tau^{-(p+1) / 2}\right) \quad \text { quand } \tau \rightarrow+\infty,
$$

où $G_{\gamma}$ est défini par (9) lorsque $\gamma$ est donné par (8).

Remarque. L'aire $(m-1)$-dimensionnelle de $S^{+}$est égale à $2 \pi^{m / 2} / \Gamma(m / 2)$, si bien que, lorsque $K^{+}=S^{+}$et $K^{-}=S^{-}$, autrement dit si $\gamma=\mathbb{R}^{p}$, alors $G_{\gamma}=1$, et on retrouve le terme principal classique. Seul le terme d'erreur $O\left(\tau^{-(p+1) / 2}\right)$ de (10) est moins bon que le terme d'erreur $O\left(\tau^{-p / 2-1}\right)$ dans $(2)$.

\section{References}

[1] J. Guo, On lattices points in large convex bodies, Acta Arithmetica 151.1 (2012), 83-108.

[2] S. Graham and G. Kolesnik, Van der Corput's Method of Exponential Sums, vol. 126 of London Mathematical Society Lecture Note Series, Cambridge University Press, 1991.

[3] L. Hörmander, The analysis of linear partial differential operators I, Springer, Berlin, 1983.

[4] M.N. Huxley, Area, lattice points and exponential sums, Clarendon Press, Oxford, 1996.

[5] E. Krätzel, Lattice points, Kluwer, 1988.

[6] E. Krätzel, Double exponential sums, Analysis 16 (1996), 109-123.

[7] S. Lang, Undergraduate analysis, Springer, 2005.

[8] H.Q. Liu, On the estimates of double exponential sums, Acta Arithmetica 129.3 (2007), 203-247.

[9] W. Müller, Lattice points in large convex bodies, Mh. Math. 128 (1999), 315330.

[10] M. Redouaby and P. Sargos, Sur la transformation B de Van der Corput, Expo. Math. 17 (1999), 207-232.

[11] B.R. Srinivasan, The lattice point problem of many-dimensional hyperbolö̈ds II, Acta Arithmetica VIII (1963), 173-204.

[12] B.R. Srinivasan, The lattice point problem of many-dimensional hyperboloids III, Math. Annalen 160 (1965), 280-311.

[13] E.M. Stein, Harmonic Analysis : real-variable methods, orthogonality, and oscillatory integrals, Princeton Mathematical Series 43, 1999.

[14] J. Vandehey, Error term improvements for Van der Corput transforms, preprint, 2012, at arXiv:1205.0090.

Received: 1 January 2014; revised: 11 April 2014 